\title{
3-Aminopyrrolidines via Ring Rearrangement of 2-Aminomethylazetidines. Synthesis of (-)-Absouline
}

Monica Vargas-Sanchez, François Couty, ${ }^{*}$ Gwilherm Evano, ${ }^{*}$ Damien Prim and Jérome Marrot

Laboratoire SIRCOB, UMR CNRS 8086, Université de Versailles Saint Quentin en Yvelines, 45 avenue des Etats-Unis, 78035 Versailles Cedex, France

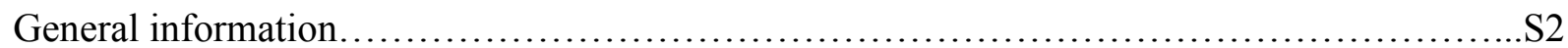

Synthesis of Azetidinic Diamines 6 via nucleophilic addition/reduction sequence...............S3

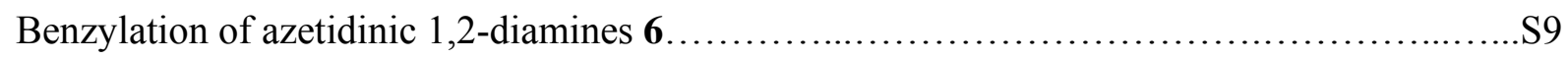

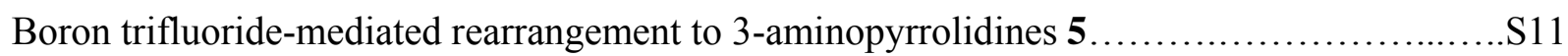

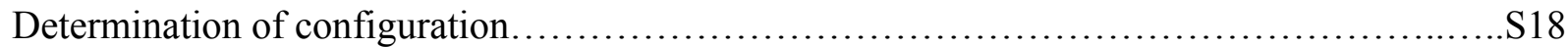

Synthesis of (-)-Absouline........................................................... 19

Absouline Spectral Data Compared to Reported Data.......................................... 25

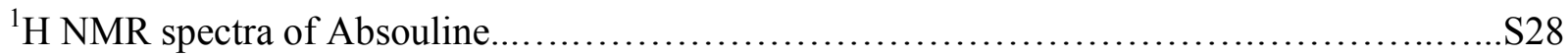

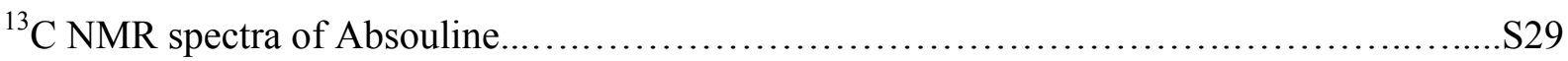

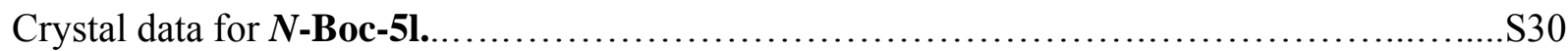


General Information. All reactions were carried out in oven or flame-dried glassware under an argon atmosphere employing standard techniques in handling air-sensitive materials. All solvents were reagent grade. Tetrahydrofuran (THF), diethyl ether $\left(\mathrm{Et}_{2} \mathrm{O}\right)$ and toluene were freshly distilled from sodium/benzophenone under argon immediately prior to use. Dichloromethane, and acetonitrile were freshly distilled from calcium hydride. $N, N$-Dimethylformamide and dimethylsulfoxide were distilled over calcium hydride and stored over $4 \AA$ molecular sieves. Triethylamine and diisopropylethylamine were distilled over calcium hydride and stored over potassium hydroxide. Benzaldehyde was distilled before use. Boron trifluoride diethyletherate was distilled immediately before use. All other reagents were used as supplied. Unless otherwise noted, reactions were magnetically stirred and monitored by thin layer chromatography with Merck-Kiesegel $60 \mathrm{~F}_{254}$ plates using mixtures of ethyl acetate (AcOEt), diethylether, methanol, petroleum ether (PE) and 30\% aqueous ammonia solution $\left(\mathrm{NH}_{3}\right)$. Flash chromatographies were performed with silica gel 60 (particle size 35-70 $\mu \mathrm{m}$ ) supplied by SDS. Yields refer to chromatographically and spectroscopically pure compounds, unless otherwise noted. Proton NMR spectra were recorded using an internal deuterium lock at ambient temperature (unless otherwise stated) on a Bruker Avance-300 spectrometer at $300 \mathrm{MHz}$. An internal reference of $\delta_{\mathrm{H}}$ 7.27 was used for $\mathrm{CDCl}_{3}$. Data are presented as follows: chemical shift (in ppm on the $\delta$ scale relative to $\left.\delta_{\mathrm{TMS}}=0\right)$, multiplicity $(\mathrm{s}=$ singlet, $\mathrm{d}=$ doublet, $\mathrm{t}=$ triplet, $\mathrm{q}=$ quartet, quint. $=$ quintet, $\mathrm{m}=$ multiplet, $\mathrm{br}=$ broad, $\mathrm{ABq}=\mathrm{AB}$ quartet $)$, coupling constant $(\mathrm{J} / \mathrm{Hz})$ and integration. Resonances that are either partially or fully obscured are denoted obscured (obsc). Carbon-13 NMR spectra were recorded on a Bruker Avance-300 spectrometer at $75 \mathrm{MHz}$. Melting points were measured on a Tottoli Büchi apparatus. An internal reference of $\delta_{\mathrm{C}} 77.16$ was used for $\mathrm{CDCl}_{3}$. Optical rotations were recorded on a Perkin Elmer 341 polarimeter at $589 \mathrm{~nm}$ and reported as follows: $[\alpha]_{\mathrm{D}}^{20}$, concentration $(c$ in $\mathrm{g} / 100 \mathrm{~mL})$ and solvent. Mass spectra were obtained on a GCMS HP MS 5989B spectrometer. High-resolution mass-spectra were obtained on a Finnagan MAT-90 spectrometer in the Boston University Mass Spectrometry Laboratory. 


\section{Experimental Procedures}

\section{Synthesis of Azetidinic Diamines 6 via nucleophilic addition/reduction sequence: general procedure A using organolithium reagents. ${ }^{\text {s1 }}$}

To a solution of cyanoazetidine $\mathrm{S}^{\mathrm{S}}(1.0 \mathrm{mmol})$ in toluene $(5 \mathrm{~mL})$ was added a solution of organolithium reagent $(2.0 \mathrm{mmol})$ dropwise at $0{ }^{\circ} \mathrm{C}$. The resulting brown solution was stirred at 0 ${ }^{\circ} \mathrm{C}$ for $15 \mathrm{~min}$ and quenched with methanol $(5 \mathrm{~mL})$. Sodium borohydride (58 mg, $1.5 \mathrm{mmol}$ ) was then added in one portion, the reaction mixture was stirred for $2 \mathrm{~h}$ at $0^{\circ} \mathrm{C}$ and carefully hydrolyzed by addition of an aqueous saturated solution of $\mathrm{NH}_{4} \mathrm{Cl}$. Methanol was evaporated under reduced pressure, $1 \mathrm{M}$ aqueous $\mathrm{NaOH}$ solution was added of and the aqueous layer was extracted with AcOEt. Combined organic extracts were washed with brine, dried over $\mathrm{MgSO}_{4}$, filtered and concentrated under vacuum. The residue was purified by column chromatography (see below for solvent mixtures) to yield the desired diamines.

\section{Synthesis of Azetidinic Diamines 6 via nucleophilic addition/reduction sequence: general procedure B using Grignard reagents.}

To a solution of cyanoazetidine $(1.0 \mathrm{mmol})$ in THF $(15 \mathrm{~mL})$ was added a solution of Grignard reagent $(4.0 \mathrm{mmol})$ dropwise at $0{ }^{\circ} \mathrm{C}$. The resulting brown solution was stirred overnight at $\mathrm{rt}$ and quenched with methanol $(10 \mathrm{~mL})$. Sodium borohydride $(58 \mathrm{mg}, 1.5 \mathrm{mmol})$ was then added in one portion, the reaction mixture was stirred for $2 \mathrm{~h}$ at $0^{\circ} \mathrm{C}$ and carefully hydrolyzed by addition of an aqueous saturated solution of $\mathrm{NH}_{4} \mathrm{Cl}$. Methanol was evaporated under reduced pressure, $1 \mathrm{M}$ aqueous $\mathrm{NaOH}$ solution was added and the aqueous layer was extracted with AcOEt. Combined organic extracts were washed with brine, dried over $\mathrm{MgSO}_{4}$, filtered and concentrated under vacuum. The residue was purified by column chromatography (see below for solvent mixtures) to yield the desired diamines.

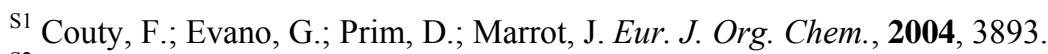

S2 Agami, C.; Couty, F.; Evano, G. Tetrahedron: Asymmetry 2002, 13, 297.
} 


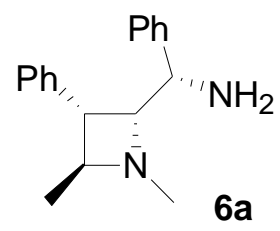

[2R,2(1S),3S,4S]-2-(Amino-phenyl-methyl)-1,4-dimethyl-3-phenyl-azetidine 6a. Procedure A; column chromatography: silica gel, AcOEt/PE: 50/50; pale yellow solid; Yield: 78 \%; $\mathrm{R}_{\mathrm{f}}$ : 0.3 $\left(\mathrm{Et}_{2} \mathrm{O} / \mathrm{PE}: 70 / 30\right) ; \mathrm{Mp}: 95{ }^{\circ} \mathrm{C} ;[\alpha]_{\mathrm{D}}^{20}:+283\left(c 0.3, \mathrm{CHCl}_{3}\right) ;{ }^{1} \mathrm{H}$ NMR $\left(300 \mathrm{MHz}, \mathrm{CDCl}_{3}\right): \delta 7.16-$ $7.54(\mathrm{~m}, 10 \mathrm{H}), 3.66-3.73(\mathrm{~m}, 2 \mathrm{H}), 3.50(\mathrm{dq}, J=9.2$ and $6.3 \mathrm{~Hz}, 1 \mathrm{H}), 3.22(\mathrm{dd}, J=5.4$ and 2.6 $\mathrm{Hz}, 1 \mathrm{H}), 1.50(\mathrm{~s}, 3 \mathrm{H}), 1.20$ (d, $J=6.6 \mathrm{~Hz}, 3 \mathrm{H}), 1.11$ (br. s, $2 \mathrm{H}) ;{ }^{13} \mathrm{C} \mathrm{NMR}\left(300 \mathrm{MHz}, \mathrm{CDCl}_{3}\right): \delta$ 143.3, 140.1, 129.1, 128.5, 128.3, 127.6, 127.4, 126.8, 74.3, 61.9, 56.6, 47.9, 36.2, 14.7; CIMS ( $\mathrm{NH}_{3}$ gas): 267.3, 250.3, $131.8 ; \mathrm{HRMS}\left(\mathrm{CI}, \mathrm{NH}_{3}\right.$ ) $m / z$ calcd for $\mathrm{C}_{18} \mathrm{H}_{23} \mathrm{~N}_{2}[\mathrm{M}+\mathrm{H}]^{+} 267.186$ found 267.183.

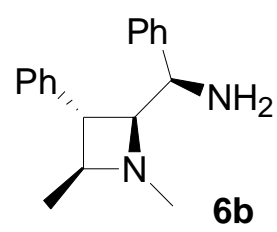

[2S,2(1R),3S,4S]-2-(Amino-phenyl-methyl)-1,4-dimethyl-3-phenyl-azetidine 6b. Procedure A; column chromatography: silica gel, AcOEt/PE: 50/50; pale yellow oil; Yield: $76 \%$; $\mathrm{R}_{\mathrm{f}}: 0.3$ $\left(\mathrm{Et}_{2} \mathrm{O} / \mathrm{PE}: 70 / 30\right) ;[\alpha]_{\mathrm{D}}^{20}:-98\left(c 1.0, \mathrm{CHCl}_{3}\right) ;{ }^{1} \mathrm{H}$ NMR $\left(300 \mathrm{MHz}, \mathrm{CDCl}_{3}\right): \delta 7.31-7.98(\mathrm{~m}, 10 \mathrm{H})$, $4.0(\mathrm{~d}, J=5.4 \mathrm{~Hz}, 1 \mathrm{H}), 3.04-3.07(\mathrm{~m}, 2 \mathrm{H}), 2.71-2.88(\mathrm{~m}, 1 \mathrm{H}), 2.01(\mathrm{~s}, 3 \mathrm{H}), 1.63$ (br. s, 2H), 1.15 $(\mathrm{d}, J=6.0 \mathrm{~Hz}, 3 \mathrm{H}) ;{ }^{13} \mathrm{C} \mathrm{NMR}\left(75 \mathrm{MHz}, \mathrm{CDCl}_{3}\right): \delta 142.7,141.1,128.3,128.2,127.5,127.2$, 127.1, 126.2, 67.9, 59.2, 47.9, 42.7, 30.9, 20.2; CIMS ( $\mathrm{NH}_{3}$ gas): 289.3, 250.3; HRMS (CI, $\left.\mathrm{NH}_{3}\right)$ $m / z$ calcd for $\mathrm{C}_{18} \mathrm{H}_{23} \mathrm{~N}_{2}[\mathrm{M}+\mathrm{H}]^{+} 267.186$ found 267.189 . 


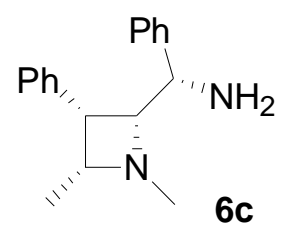

[2R,2(1S),3S,4R]-2-(Amino-phenyl-methyl)-1,4-dimethyl-3-phenyl-azetidine 6c. Procedure A; column chromatography: silica gel, AcOEt/EtOH/NH 3 : 90/7/3; pale yellow oil; Yield: $88 \%$; $\mathrm{R}_{\mathrm{f}}$ : $0.3\left(\mathrm{AcOEt} / \mathrm{EtOH} / \mathrm{NH}_{3}: 90 / 9 / 1\right) ;[\alpha]_{\mathrm{D}}^{20}:+36\left(c 0.5, \mathrm{CHCl}_{3}\right) ;{ }^{1} \mathrm{H} \mathrm{NMR}\left(300 \mathrm{MHz}, \mathrm{CDCl}_{3}\right): \delta 7.04-$ $7.44(\mathrm{~m}, 10 \mathrm{H}), 4.17(\mathrm{~d}, J=6.4 \mathrm{~Hz}, 1 \mathrm{H}), 3.98-4.11(\mathrm{~m}, 2 \mathrm{H}), 3.72(\mathrm{dd}, J=7.9$ and $6.6 \mathrm{~Hz}, 1 \mathrm{H})$, 1.93 (s, 3H), 1.83 (br. s, $2 \mathrm{H}), 0.78$ (d, $J=6.3 \mathrm{~Hz}, 3 \mathrm{H}) ;{ }^{13} \mathrm{C} \mathrm{NMR}\left(75 \mathrm{MHz}, \mathrm{CDCl}_{3}\right): \delta 143.0$, 138.0, 128.3, 128.1, 127.9, 127.3, 127.2, 126.0, 73.7, 59.9, 59.8, 43.0, 35.6, 10.9; CIMS $\left(\mathrm{NH}_{3}\right.$ gas): 267, 248, 147; HRMS (CI, $\left.\mathrm{NH}_{3}\right) \mathrm{m} / z$ calcd for $\mathrm{C}_{18} \mathrm{H}_{23} \mathrm{~N}_{2}[\mathrm{M}+\mathrm{H}]^{+} 267.186$ found 267.189

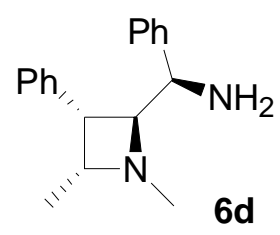

[2S,2(1R),3S,4R]-2-(Amino-phenyl-methyl)-1,4-dimethyl-3-phenyl-azetidine 6d. Procedure A; column chromatography: silica gel, AcOEt/PE: 70/30; pale yellow oil; Yield: $33 \%$; $\mathrm{R}_{\mathrm{f}}: 0.3$ $\left(\mathrm{Et}_{2} \mathrm{O} / \mathrm{PE}: 70 / 30\right) ;[\alpha]_{\mathrm{D}}^{20}:-199\left(c 0.3, \mathrm{CHCl}_{3}\right) ;{ }^{1} \mathrm{H}$ NMR $\left(300 \mathrm{MHz}, \mathrm{CDCl}_{3}\right): \delta 7.61-7.66(\mathrm{~m}, 2 \mathrm{H}$, CHAr), 7.14-7.36 (m, 8H), $3.95(\mathrm{~d}, J=9.6 \mathrm{~Hz}, 1 \mathrm{H}), 3.62(\mathrm{dd}, J=15.2$ and $7.7 \mathrm{~Hz}, 1 \mathrm{H}), 3.07-3.16$ (m, 2H), 1.53 (s, 3H), 1.27 (br. s, 2H), 0.88 (d, $J=6.4 \mathrm{~Hz}, 3 \mathrm{H}) ;{ }^{13} \mathrm{C}$ NMR $\left(75 \mathrm{MHz}, \mathrm{CDCl}_{3}\right): \delta$ $142.9,136.7,131.0,128.4,128.0,127.6,127.4,126.8,75.5,63.6,55.1,45.7,43.3$, 14.4; CIMS $\left(\mathrm{NH}_{3}\right.$ gas): 279, 267, 160; HRMS (CI, $\left.\mathrm{NH}_{3}\right) \mathrm{m} / z$ calcd for $\mathrm{C}_{18} \mathrm{H}_{23} \mathrm{~N}_{2}[\mathrm{M}+\mathrm{H}]^{+} 267.186$ found 267.186. 


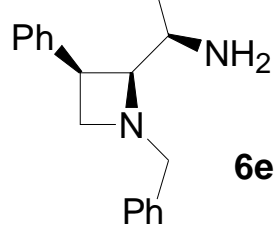

[2S,2(1R),3R]-2-(1-Amino-ethyl)-1-benzyl-3-phenyl-azetidine 6e. Procedure $\mathrm{B}$; column chromatography: silica gel, AcOEt/ $\mathrm{NH}_{3}$ : 99/1, then AcOEt/EtOH/ $\mathrm{NH}_{3}: 90 / 9 / 1$; colorless oil; Yield: $48 \%$; $\mathrm{R}_{\mathrm{f}}: 0.1$ (AcOEt/EtOH/ $\left.\mathrm{NH}_{3}: 90 / 9.5 / 0.5\right) ;[\alpha]_{\mathrm{D}}^{20}:+42\left(c \quad 0.5, \mathrm{CHCl}_{3}\right) ;{ }^{1} \mathrm{H}$ NMR (300 $\left.\mathrm{MHz}, \mathrm{CDCl}_{3}\right): \delta 7.15-7.50(\mathrm{~m}, 10 \mathrm{H}), 4.01(\mathrm{~A}$ of ABq, $J=13.4 \mathrm{~Hz}, 1 \mathrm{H}), 3.55(\mathrm{td}, J=8.3$ and 2.3 $\mathrm{Hz}, 1 \mathrm{H}$ ), 3.36 (B of ABq, $J=13.4 \mathrm{~Hz}, 1 \mathrm{H}$ ), 3.30 (A' of A'B'X syst., $J=7.7$ and $2.4 \mathrm{~Hz}, 1 \mathrm{H}$ ), 3.09-3.15 (m, 2H), 2.75 (m, 1H), 1.74 (br. s, 2H), 0.96 (d, $J=6.4 \mathrm{~Hz}, 3 \mathrm{H}) ;{ }^{13} \mathrm{C} \mathrm{NMR}(75 \mathrm{MHz}$, $\left.\mathrm{CDCl}_{3}\right): \delta 140.6,138.6,129.0,128.4,128.3,128.2,126.9,126.7,75.2,63.3,57.6,47.7,38.5$, 19.7; CIMS ( $\mathrm{NH}_{3}$ gas): 267.3, 250.3, 160.2; HRMS (CI, $\left.\mathrm{NH}_{3}\right) \mathrm{m} / z$ calcd for $\mathrm{C}_{18} \mathrm{H}_{23} \mathrm{~N}_{2}[\mathrm{M}+\mathrm{H}]^{+}$ 267.186 found 267.193 .

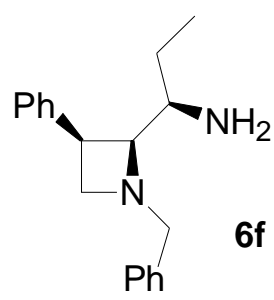

[2S,2(1R),3R]-2-(1-Amino-propyl)-1-benzyl-3-phenyl-azetidine 6f. Procedure B; column chromatography: silica gel, AcOEt/EtOH/ $/ \mathrm{NH}_{3}$ : 90/9.7/0.3; colorless oil; Yield: $34 \%$; $\mathrm{R}_{\mathrm{f}}: 0.2$ $\left(\mathrm{AcOEt} / \mathrm{EtOH} / \mathrm{NH}_{3}: 90 / 9.5 / 0.5\right) ;[\alpha]_{\mathrm{D}}^{20}:+16\left(c 1.3, \mathrm{CHCl}_{3}\right) ;{ }^{1} \mathrm{H} \mathrm{NMR}\left(300 \mathrm{MHz}, \mathrm{CDCl}_{3}\right): \delta 7.51-$ $7.44(\mathrm{~m}, 10 \mathrm{H}), 4.01$ (A of ABq, $J=13.3 \mathrm{~Hz}, 1 \mathrm{H}), 3.63(\mathrm{td}, J=8.3$ and $2.8 \mathrm{~Hz}, 1 \mathrm{H}), 3.34$ (B of ABq, $J=13.3 \mathrm{~Hz}, 1 \mathrm{H}$ ), 3.29 (A' of A'B'X syst., $J=7.1$ and $2.5 \mathrm{~Hz}, 1 \mathrm{H}), 3.08-3.21(\mathrm{~m}, 2 \mathrm{H}), 2.53$ $(\mathrm{td}, J=9.1$ and $3.4 \mathrm{~Hz}, 1 \mathrm{H}), 1.51-1.60(\mathrm{~m}, 1 \mathrm{H}), 1.37$ (br. s, $2 \mathrm{H}), 1.01-1.18(\mathrm{~m}, 1 \mathrm{H}), 0.80(\mathrm{t}, J=$ $7.5 \mathrm{~Hz}, 3 \mathrm{H}) ;{ }^{13} \mathrm{C} \mathrm{NMR}\left(75 \mathrm{MHz}, \mathrm{CDCl}_{3}\right): \delta 140.1,139.8,129.4,129.1,128.4,128.3,128.1$, 126.8, 73.7, 63.5, 57.7, 53.2, 38.5, 25.7, 9.9; CIMS ( $\mathrm{NH}_{3}$ gas): 281.3, 264.3; HRMS (CI, $\mathrm{NH}_{3}$ ) $m / z$ calcd for $\mathrm{C}_{19} \mathrm{H}_{25} \mathrm{~N}_{2}[\mathrm{M}+\mathrm{H}]^{+} 281.202$ found 281.206. 


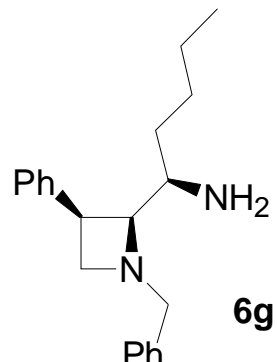

$\mathrm{Ph}$

[2S,2(1R),3R]-2-(1-Amino-pentyl)-1-benzyl-3-phenyl-azetidine 6g. Procedure A; column chromatography: silica gel, $\mathrm{Et}_{2} \mathrm{O} / \mathrm{PE} / \mathrm{NH}_{3}: 60 / 35 / 5$; colorless oil; Yield: $48 \%$; $\mathrm{R}_{\mathrm{f}}$ : 0.1 $\left(\mathrm{AcOEt} / \mathrm{EtOH} / \mathrm{NH}_{3}: 90 / 9.5 / 0.5\right) ;[\alpha]_{\mathrm{D}}^{20}:+49\left(c\right.$ 0.4, $\left.\mathrm{CHCl}_{3}\right) ;{ }^{1} \mathrm{H} \mathrm{NMR}\left(300 \mathrm{MHz}, \mathrm{CDCl}_{3}\right): \delta 7.44-$ $7.47(\mathrm{~m}, 2 \mathrm{H}), 7.24-7.32(\mathrm{~m}, 6 \mathrm{H}), 7.13-7.20(\mathrm{~m}, 2 \mathrm{H}), 4.01$ (A of ABq, $J=13.3 \mathrm{~Hz}, 1 \mathrm{H}), 3.53(\mathrm{td}$, $J=8.3$ and $2.6 \mathrm{~Hz}, 1 \mathrm{H}$ ), 3.37 (B of ABq, $J=13.7 \mathrm{~Hz}, 1 \mathrm{H}$ ), 3.29 (A' of A'B'X syst., $J=7.7$ and $2.7 \mathrm{~Hz}, 1 \mathrm{H}), 3.09-3.19(\mathrm{~m}, 2 \mathrm{H}), 2.59(\mathrm{td}, J=9.1$ and $3.7 \mathrm{~Hz}, 1 \mathrm{H}), 1.42-1.51(\mathrm{~m}, 1 \mathrm{H}), 0.99-1.30$ $(\mathrm{m}, 5 \mathrm{H}), 0.77(\mathrm{t}, J=6.9 \mathrm{~Hz}, 3 \mathrm{H}) ;{ }^{13} \mathrm{C} \mathrm{NMR}\left(75 \mathrm{MHz}, \mathrm{CDCl}_{3}\right): \delta 140.8,138.7,129.1,128.4$, $128.3,128.2,128.1,126.9,74.3,63.7,57.8,52.0,38.6,33.0,27.9,22.9,14.0 ; \mathrm{CIMS}\left(\mathrm{NH}_{3}\right.$ gas $)$ : 308, 189; HRMS (CI, $\mathrm{NH}_{3}$ ) $m / z$ calcd for $\mathrm{C}_{21} \mathrm{H}_{29} \mathrm{~N}_{2}[\mathrm{M}+\mathrm{H}]^{+} 309.233$ found 309.238.

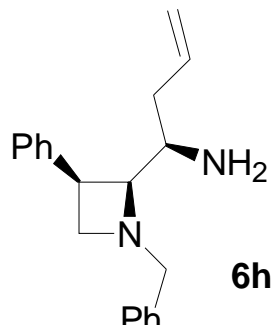

[2S,2(1R),3R]-2-(1-Amino-but-3-enyl)-1-benzyl-3-phenyl-azetidine 6h. Procedure B; column chromatography: silica gel, AcOEt/EtOH/ $/ \mathrm{NH}_{3}: 95 / 4.5 / 0.5$; white solid; Yield: $86 \%$; $\mathrm{R}_{\mathrm{f}}: 0.7$ (ACOEt/MeOH/NH $3:$ 90/9.7/0.3); Mp: $72{ }^{\circ} \mathrm{C} ;[\alpha]_{\mathrm{D}}^{20}:+14\left(c \quad 0.45, \mathrm{CHCl}_{3}\right) ;{ }^{1} \mathrm{H}$ NMR $(300 \mathrm{MHz}$, $\left.\mathrm{CDCl}_{3}\right): \delta 7.13-7.47(\mathrm{~m}, 10 \mathrm{H}), 5.55-5.67(\mathrm{~m}, 1 \mathrm{H}), 4.93-4.99(\mathrm{~m}, 2 \mathrm{H}), 4.01$ (A of ABq, $J=13.3$ $\mathrm{Hz}, 1 \mathrm{H}$ ), 3.55 (td, $J=8.3$ and $2.5 \mathrm{~Hz}, 1 \mathrm{H}$ ), 3.36 (B of ABq, $J=13.3 \mathrm{~Hz}, 1 \mathrm{H}$ ), 3.31 (app. d, $J=$ $7.7 \mathrm{~Hz}, 1 \mathrm{H}$ ), 3.20 (app. t, $J=8.3 \mathrm{~Hz}, 1 \mathrm{H}$ ), 3.11 (app. t, $J=7.9 \mathrm{~Hz}, 1 \mathrm{H}$ ), 2.68 (td, $J=9.2$ and 3.3 $\mathrm{Hz}, 1 \mathrm{H}), 2.28-2.35(\mathrm{~m}, 1 \mathrm{H}), 1.72-1.82(\mathrm{~m}, 1 \mathrm{H}), 0.90$ (br. s, $2 \mathrm{H}) ;{ }^{13} \mathrm{C} \mathrm{NMR}\left(75 \mathrm{MHz}, \mathrm{CDCl}_{3}\right): \delta$ 140.6, 138.5, 135.3, 129.1, 128.5, 128.4, 127.0, 126.8, 117.8, 73.7, 63.6, 57.8, 51.1, 38.5, 37.9; CIMS (electrospray): 315.3, 293.3, 276.3; HRMS (CI, $\mathrm{NH}_{3}$ ) $m / z$ calcd for $\mathrm{C}_{20} \mathrm{H}_{25} \mathrm{~N}_{2}[\mathrm{M}+\mathrm{H}]^{+}$ 293.202 found 293.197. 


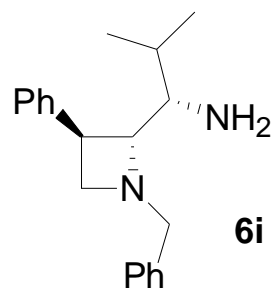

[2R,2(1S),3R]-2-(1-Amino-2-methyl-propyl)-1-benzyl-3-phenyl-azetidine 6i. Procedure B; column chromatography: silica gel, AcOEt/ $\mathrm{NH}_{3}$ : 99/1 then AcOEt/EtOH/ $/ \mathrm{NH}_{3}$ : 90/9/1; colorless oil; Yield: 50 \%; Rf: 0.2 (AcOEt/EtOH/NH $\left./ \mathrm{NH}_{3}: 90 / 9.5 / 0.5\right) ;[\alpha]_{\mathrm{D}}^{20}:+40\left(\right.$ c 1.2, $\left.\mathrm{CHCl}_{3}\right) ;{ }^{1} \mathrm{H}$ NMR (300 MHz, $\left.\mathrm{CDCl}_{3}\right): \delta 7.10-7.30(\mathrm{~m}, 10 \mathrm{H}), 3.77$ (A of ABq, $\left.J=12.7 \mathrm{~Hz}, 1 \mathrm{H}\right), 3.57-3.63(\mathrm{~m}, 3 \mathrm{H})$, 3.47 (m, 1H), 2.89 (m, 1H), 2.44 (br. s, 2H), 2.32 (dd, $J=7.7$ and $3.9 \mathrm{~Hz}, 1 \mathrm{H}), 1.42(\mathrm{~m}, 1 \mathrm{H})$, $0.78(\mathrm{~d}, J=6.7 \mathrm{~Hz}, 3 \mathrm{H}), 0.50(\mathrm{~d}, J=6.7 \mathrm{~Hz}, 3 \mathrm{H}) ;{ }^{13} \mathrm{C} \mathrm{NMR}\left(75 \mathrm{MHz}, \mathrm{CDCl}_{3}\right): \delta 141.7,138.2$, $128.7,128.4,128.3,128.0,127.2,126.5,75.1,62.0,59.1,58.3,30.9,29.6,19.5,19.4$; CIMS $\left(\mathrm{NH}_{3}\right.$ gas): 295.3, 278.3; HRMS (CI, $\left.\mathrm{NH}_{3}\right) \mathrm{m} / z$ calcd for $\mathrm{C}_{20} \mathrm{H}_{27} \mathrm{~N}_{2}[\mathrm{M}+\mathrm{H}]^{+} 295.217$ found 295.217.

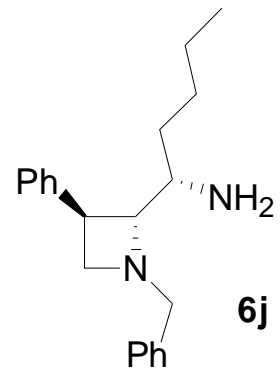

[2R,2(1S),3R]-2-(1-Amino-pentyl)-1-benzyl-3-phenyl-azetidine 6j. Procedure A; column chromatography: silica gel, AcOEt/ $\mathrm{NH}_{3}$ : 99/1, then AcOEt/EtOH/ $/ \mathrm{NH}_{3}: 90 / 9 / 1$; colorless oil; Yield: 65 \%; Rf: 0.2 (AcOEt/EtOH/NH $/ \mathrm{NH}_{3}$ 90/9.5/0.5); $[\alpha]_{\mathrm{D}}^{20}:+22\left(c\right.$ 1.0, $\left.\mathrm{CHCl}_{3}\right) ;{ }^{1} \mathrm{H}$ NMR (300 $\left.\mathrm{MHz}, \mathrm{CDCl}_{3}\right): \delta 7.06-8.05(\mathrm{~m}, 10 \mathrm{H}), 3.76(\mathrm{~A}$ of ABq, $J=12.7 \mathrm{~Hz}, 1 \mathrm{H}), 3.49-3.62(\mathrm{~m}, 3 \mathrm{H}), 3.28$ (dd, $J=7.5$ and $4.0 \mathrm{~Hz}, 1 \mathrm{H}), 2.86(\mathrm{td}, J=6.0$ and $1.7 \mathrm{~Hz}, 1 \mathrm{H}), 2.57-2.63(\mathrm{~m}, 1 \mathrm{H}), 2.0$ (br. s, 2H), $0.95-1.19(\mathrm{~m}, 6 \mathrm{H}), 0.66(\mathrm{t}, J=6.7 \mathrm{~Hz}, 3 \mathrm{H}) ;{ }^{13} \mathrm{C} \mathrm{NMR}\left(75 \mathrm{MHz}, \mathrm{CDCl}_{3}\right): \delta 142.0,138.4,128.7$, $128.4,128.3,128.1,127.9,126.4,77.3,62.4,59.0,52.2,36.5,32.5,28.3,22.6,13.9 ; \mathrm{CIMS}\left(\mathrm{NH}_{3}\right.$ gas): 308, 189; HRMS (CI, $\left.\mathrm{NH}_{3}\right) m / z$ calcd for $\mathrm{C}_{21} \mathrm{H}_{29} \mathrm{~N}_{2}[\mathrm{M}+\mathrm{H}]^{+} 309.233$ found 309.227. 


\section{Benzylation of azetidinic 1,2-diamines 6; general procedure.}

A solution of diamine $(1.0 \mathrm{mmol})$ and benzaldehyde $(100 \mu \mathrm{L}, 1.0 \mathrm{mmol})$ in DCM $(3 \mathrm{~mL})$ was treated with $\mathrm{MgSO}_{4}(\sim 200 \mathrm{mg})$. The reaction mixture was left at $\mathrm{rt}$ for one hour without stirring, filtered and concentrated. The residue was dissolved in methanol $(3 \mathrm{~mL})$ and sodium borohydride (45 mg, $1.2 \mathrm{mmol}$ ) was added at $0{ }^{\circ} \mathrm{C}$. The resulting mixture was stirred for $2 \mathrm{~h}$ and quenched by addition of a saturated aqueous solution of $\mathrm{NH}_{4} \mathrm{Cl}$. Methanol was evaporated under reduced pressure, $1 \mathrm{M}$ aqueous $\mathrm{NaOH}$ solution was added of and the aqueous layer was extracted with AcOEt. Combined organic extracts were washed with brine, dried over $\mathrm{MgSO}_{4}$, filtered and concentrated under vacuum. The residue was purified by column chromatography (see below for solvent mixtures) to yield the desired benzylated diamines.

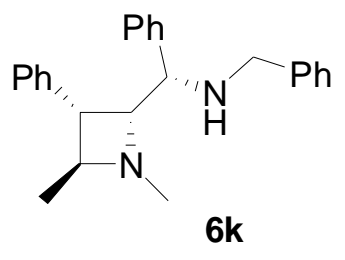

[2R,2(1S),3S,4S]-2-(Benzylamino-phenyl-methyl)-1,4-dimethyl-3-phenyl-azetidine

$6 \mathbf{k}$.

Column chromatography: silica gel, $\mathrm{Et}_{2} \mathrm{O} / \mathrm{PE} / \mathrm{NH}_{3}: 70 / 29.5 / 0.5$; colorless oil; Yield: $92 \%$; $\mathrm{R}_{\mathrm{f}}: 0.3$ $\left(\mathrm{Et}_{2} \mathrm{O} / \mathrm{PE} / \mathrm{NH}_{3}: 70 / 29.5 / 0.5\right) ;[\alpha]_{\mathrm{D}}^{20}:-34\left(c 0.6, \mathrm{CHCl}_{3}\right) ;{ }^{1} \mathrm{H} \mathrm{NMR}\left(300 \mathrm{MHz}, \mathrm{CDCl}_{3}\right): \delta 7.43-7.46$ (m, 2H), 7.13-7.32 (m, 8H), 7.01-7.03 (m, 3H), 6.59-6.6 (m, 2H), 3.69-3.71 (br. m, 1H), 3.52 (app. t, $J=9.0 \mathrm{~Hz}, 1 \mathrm{H}), 3.49$ (d, $J=9.0 \mathrm{~Hz}, 1 \mathrm{H}), 3.10-3.15(\mathrm{~m}, 2 \mathrm{H}), 2.66$ (B of ABq, $J=12.6$ $\mathrm{Hz}, 1 \mathrm{H}), 1.44(\mathrm{~s}, 3 \mathrm{H}), 1.17(\mathrm{~d}, J=6.6 \mathrm{~Hz}, 3 \mathrm{H}) ;{ }^{13} \mathrm{C} \mathrm{NMR}\left(75 \mathrm{MHz}, \mathrm{CDCl}_{3}\right): \delta 141.2,140.0$, 139.8, 129.1, 128.6, 128.4, 128.2, 127.6, 127.3, 127.0, 126.9, 126.5, 73.0, 63.7, 62.0, 50.9, 47.9, 36.4, 14.8; CIMS ( $\mathrm{NH}_{3}$ gas): 357.4, 250.3, 239.3, 131.9; HRMS (CI, $\mathrm{NH}_{3}$ ) m/z calcd for $\mathrm{C}_{25} \mathrm{H}_{29} \mathrm{~N}_{2}[\mathrm{M}+\mathrm{H}]^{+} 357.233$ found 357.234 . 


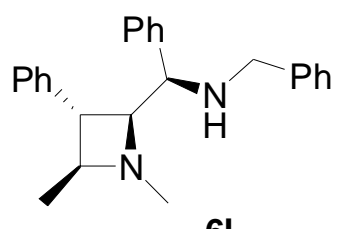

6l

[2S,2(1R),3S,4S]-2-(Benzylamino-phenyl-methyl)-1,4-dimethyl-3-phenyl-azetidine

Column chromatography: silica gel, $\mathrm{Et}_{2} \mathrm{O} / \mathrm{PE} / \mathrm{NH}_{3}: 70 / 29.5 / 0.5$; colorless oil; Yield: $87 \%$; $\mathrm{R}_{\mathrm{f}}: 0.3$ $\left(\mathrm{Et}_{2} \mathrm{O} / \mathrm{PE} / \mathrm{NH}_{3}: 70 / 29.5 / 0.5\right) ;[\alpha]_{\mathrm{D}}^{20}:-71\left(c 0.5, \mathrm{CHCl}_{3}\right) ;{ }^{1} \mathrm{H} \mathrm{NMR}\left(300 \mathrm{MHz}, \mathrm{CDCl}_{3}\right): \delta 6.97-7.41$ (m, 15H), 3.80-3.81 (m, 1H), 3.78 (A of ABq, $J=13.5 \mathrm{~Hz}, 1 \mathrm{H}), 3.53$ (B of ABq, $J=13.8 \mathrm{~Hz}$, 1H), 3.13-3.24 (m, 2H), 2.78 (app. quint., $J=6.2 \mathrm{~Hz}, 1 \mathrm{H}$ ), 2.21 (br. s, 1H), 2.10 (s, 3H), 1.23 (d, $J=6.0 \mathrm{~Hz}, 3 \mathrm{H}) ;{ }^{13} \mathrm{C} \mathrm{NMR}\left(75 \mathrm{MHz}, \mathrm{CDCl}_{3}\right): \delta 141.2,140.9,140.7,128.6,128.3,128.2,128.0$, 127.9, 127.5, 127.1, 126.5, 126.1, 75.9, 67.8, 64.2, 50.8, 47.7, 42.4, 20.1; CIMS $\left(\mathrm{NH}_{3}\right.$ gas): 357.4, 239.3, 131.9; HRMS (CI, $\mathrm{NH}_{3}$ ) $m / z$ calcd for $\mathrm{C}_{25} \mathrm{H}_{29} \mathrm{~N}_{2}[\mathrm{M}+\mathrm{H}]^{+} 357.233$ found 357.229. 


\section{Boron trifluoride mediated rearrangement to 3-aminopyrrolidines 5; general procedure.}

To a stirred solution of 1,2 diamine $6(0.2 \mathrm{mmol})$ in acetonitrile $(1 \mathrm{~mL})$ was added $\mathrm{BF}_{3} \cdot \mathrm{OEt}_{2}(25$ $\mu \mathrm{L}, 0.2 \mathrm{mmol}$ ) at room temperature. The reaction mixture was stirred at $\mathrm{rt}$ for 1 hour and refluxed overnight. An aqueous saturated solution of $\mathrm{NaHCO}_{3}(5 \mathrm{~mL})$ was then added and the mixture was vigorously stirred at room temperature for $5 \mathrm{~min}$ before extracting the aqueous layer with AcOEt $(4 \times 5 \mathrm{~mL})$. Combined organic extracts were dried over $\mathrm{MgSO}_{4}$, filtered and concentrated under vacuum. The residue was purified by column chromatography (see below for solvent mixtures) to yield the desired 3-aminopyrrolidine.

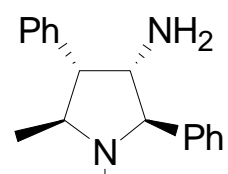

$5 a$

(2R,3S,4R,5S)-3-Amino-1,5-Dimethyl-2,4-diphenyl-pyrrolidine 5a. Column chromatography: silica gel, Et $t_{2} \mathrm{O} / \mathrm{PE}: 70 / 30$; Colorless oil; Yield: $77 \%$; $\mathrm{R}_{\mathrm{f}}: 0.3\left(\mathrm{Et}_{2} \mathrm{O} / \mathrm{PE}: 70 / 30\right) ;[\alpha]_{\mathrm{D}}^{20}:-5(c 0.5$, $\left.\mathrm{CHCl}_{3}\right) ;{ }^{1} \mathrm{H}$ NMR $\left(300 \mathrm{MHz}, \mathrm{CDCl}_{3}\right): \delta 7.15-7.39(\mathrm{~m}, 10 \mathrm{H}), 3.31(\mathrm{dd}, J=9.0$ and $6.9 \mathrm{~Hz}, 1 \mathrm{H})$, 3.05-3.16 (m, 2H), 2.78-2.92 (m, 1H), $2.10(\mathrm{~s}, 3 \mathrm{H}), 1.12(\mathrm{~d}, J=6 \mathrm{~Hz}, 3 \mathrm{H}), 0.91$ (br. s, $2 \mathrm{H}) ;{ }^{13} \mathrm{C}$ NMR (75 MHz, $\left.\mathrm{CDCl}_{3}\right): \delta 142.4,138.9,129.8,128.5,128.4,127.7,127.3,126.9,81.3,65.6$, 61.5, 56.6, 38.5, 18.1; CIMS (electrospray): 289.3, 267.3, 250.3; HRMS (CI, $\mathrm{NH}_{3}$ ) $\mathrm{m} / z$ calcd for $\mathrm{C}_{18} \mathrm{H}_{23} \mathrm{~N}_{2}[\mathrm{M}+\mathrm{H}]^{+} 267.186$ found 267.186. 


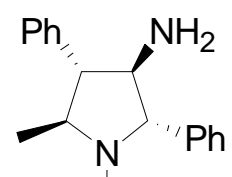

5b

(2S,3R,4R,5S)-3-Amino-1,5-dimethyl-2,4-diphenyl-pyrrolidine 5b. Column chromatography: silica gel, $\mathrm{Et}_{2} \mathrm{O} / \mathrm{PE}$ : 70/30; Colorless oil; Yield: $67 \%$; $\mathrm{R}_{\mathrm{f}}: 0.1\left(\mathrm{Et}_{2} \mathrm{O} / \mathrm{PE} / \mathrm{NH}_{3}: 80 / 19.5 / 0.5\right) ;[\alpha]_{\mathrm{D}}^{20}$ : $+39\left(c \mathrm{1.4}, \mathrm{CHCl}_{3}\right) ;{ }^{1} \mathrm{H}$ NMR $\left(300 \mathrm{MHz}, \mathrm{CDCl}_{3}\right): \delta$ 7.20-7.46 (m, 10H), 3.55-3.62 (m, 2H), 3.35 (t, $J=8.4 \mathrm{~Hz}, 1 \mathrm{H}), 2.59(\mathrm{dd}, J=8.7$ and $6.3 \mathrm{~Hz}, 1 \mathrm{H}), 2.20(\mathrm{~s}, 3 \mathrm{H}), 1.53$ (br. s, 2H), 1.25 (d, $J=$ $7.2 \mathrm{~Hz}, 3 \mathrm{H}) ;{ }^{13} \mathrm{C} \mathrm{NMR}\left(75 \mathrm{MHz}, \mathrm{CDCl}_{3}\right): \delta 142.8,141.4,128.7,128.5,128.2,128.0,127.8$, 127.6, 76.2, 67.9, 64.7, 62.4, 34.5, 16.3; CIMS (electrospray): 289.3, 250.3; HRMS (CI, $\mathrm{NH}_{3}$ ) $m / z$ calcd for $\mathrm{C}_{18} \mathrm{H}_{23} \mathrm{~N}_{2}[\mathrm{M}+\mathrm{H}]^{+} 267.186$ found 267.186 .

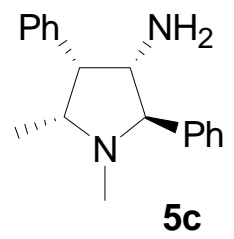

(2R,3S,4R,5R)-3-Amino-1,5-dimethyl-2,4-diphenyl-pyrrolidine 5c. Column chromatography: silica gel, $\mathrm{Et}_{2} \mathrm{O} / \mathrm{EtOH} / \mathrm{NH}_{3}$ : 90/9/1; Colorless oil; Yield: $67 \%$; $\mathrm{R}_{\mathrm{f}}$ : $0.3\left(\mathrm{Et}_{2} \mathrm{O} / \mathrm{EtOH} / \mathrm{NH}_{3}\right.$ : 90/9.5/0.5); $[\alpha]_{\mathrm{D}}^{20}:+71\left(c 0.5, \mathrm{CHCl}_{3}\right) ;{ }^{1} \mathrm{H} \mathrm{NMR}\left(300 \mathrm{MHz}, \mathrm{CDCl}_{3}\right): \delta 7.23-7.52(\mathrm{~m}, 10 \mathrm{H}), 3.39$ (app. t, $J=7.2 \mathrm{~Hz}, 1 \mathrm{H}), 2.88-3.09$ (m, 3H), 2.13 (s, 3H), 1.61 (br. s, 2H), 0.79 (d, $J=6.1 \mathrm{~Hz}$, $3 \mathrm{H}) ;{ }^{13} \mathrm{C} \mathrm{NMR}\left(75 \mathrm{MHz}, \mathrm{CDCl}_{3}\right.$ ): $\delta 141.7,141.3,129.4,128.6,128.1,127.8,127.6,126.4,80.4$, 66.7, 63.8, 56.9, 38.9, 16.8; CIMS ( $\mathrm{NH}_{3}$ gas): 289.3, 267.3, 250.2, 148.0, 131.8, 116.7; HRMS (CI, $\mathrm{NH}_{3}$ ) $m / z$ calcd for $\mathrm{C}_{18} \mathrm{H}_{23} \mathrm{~N}_{2}[\mathrm{M}+\mathrm{H}]^{+} 267.186$ found 267.183 . 


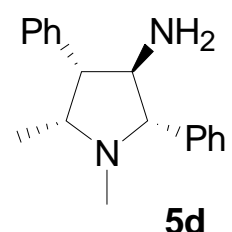

(2S,3R,4R,5R)-3-Amino-1,5-dimethyl-2,4-diphenyl-pyrrolidine 5d. Column chromatography: silica gel, $\mathrm{Et}_{2} \mathrm{O} / \mathrm{PE}: 70 / 30$ then $\mathrm{Et}_{2} \mathrm{O} / \mathrm{NH}_{3}$ : 99/1; Colorless oil; Yield: $68 \%$; $\mathrm{R}_{\mathrm{f}}: 0.2$ $\left(\mathrm{Et}_{2} \mathrm{O} / \mathrm{PE} / \mathrm{NH}_{3}: 70 / 29 / 1\right) ;[\alpha]_{\mathrm{D}}^{20}:+71\left(c 0.5, \mathrm{CHCl}_{3}\right) ;{ }^{1} \mathrm{H}$ NMR $\left(300 \mathrm{MHz}, \mathrm{CDCl}_{3}\right): \delta 7.17-7.37(\mathrm{~m}$, $10 \mathrm{H}), 3.84(\mathrm{~d}, J=7.4 \mathrm{~Hz}, 1 \mathrm{H}), 3.62-3.68(\mathrm{~m}, 2 \mathrm{H}), 3.37(\mathrm{dd}, J=7.2$ and $5.5 \mathrm{~Hz}, 1 \mathrm{H}), 2.10(\mathrm{~s}, 3 \mathrm{H})$, 1.6 (br. s, $2 \mathrm{H}), 0.90(\mathrm{~d}, J=6.4 \mathrm{~Hz}, 3 \mathrm{H}) ;{ }^{13} \mathrm{C} \mathrm{NMR}\left(75 \mathrm{MHz}, \mathrm{CDCl}_{3}\right): \delta 141.6,131.2,129.1$, 128.6, 128.5, 128.3, 127.7, 126.9, 76.2, 63.5, 62.6, 55.3, 35.2, 15.3; CIMS ( $\mathrm{NH}_{3}$ gas): 267, 160, 106; HRMS (CI, $\mathrm{NH}_{3}$ ) $m / z$ calcd for $\mathrm{C}_{18} \mathrm{H}_{23} \mathrm{~N}_{2}[\mathrm{M}+\mathrm{H}]^{+} 267.186$ found 267.183 .

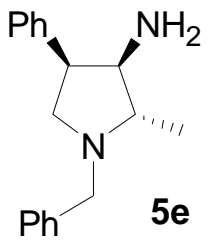

(2S,3R,4R)-3-Amino-1-benzyl-2-methyl-4-phenyl-pyrrolidine 5e. Column chromatography: silica gel, AcOEt/EtOH/NH $\mathrm{NH}_{3}$ 90/9/1; colorless oil; Yield: $69 \%$; $\mathrm{R}_{\mathrm{f}}: 0.2\left(\mathrm{AcOEt} / \mathrm{EtOH} / \mathrm{NH}_{3}\right.$ : 90/9/1); $[\alpha]_{\mathrm{D}}^{20}:+144\left(c 0.5, \mathrm{CHCl}_{3}\right) ;{ }^{1} \mathrm{H}$ NMR $\left(300 \mathrm{MHz}, \mathrm{CDCl}_{3}\right): \delta 7.04-7.30(\mathrm{~m}, 10 \mathrm{H}), 4.01$ (B of ABq, $J=12.9 \mathrm{~Hz}, 1 \mathrm{H}), 3.30-3.42$ (m, 2H), 3.10-3.17 (m, 2H), 2.60 (A' of A'B'X syst., $J=$ 10.0 and $5.0 \mathrm{~Hz}, 1 \mathrm{H}), 2.31$ (app. quint., $J=6.0 \mathrm{~Hz}, 1 \mathrm{H}), 1.24(\mathrm{~d}, J=6.2 \mathrm{~Hz}, 3 \mathrm{H}) ;{ }^{13} \mathrm{C} \mathrm{NMR}(75$ $\left.\mathrm{MHz}, \mathrm{CDCl}_{3}\right): \delta 139.4,138.8,129.2,129.1,128.5,128.3,127.1,126.6,68.3,61.4,58.5,57.2$, 47.2, 18.5; CIMS ( $\mathrm{NH}_{3}$ gas): 279, 267, 249, 147, 91; HRMS (CI, $\left.\mathrm{NH}_{3}\right) \mathrm{m} / z$ calcd for $\mathrm{C}_{18} \mathrm{H}_{23} \mathrm{~N}_{2}$ $[\mathrm{M}+\mathrm{H}]^{+} 267.186$ found 267.184 . 


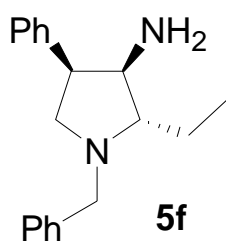

(2S,3R,4R)-3-Amino-1-benzyl-2-ethyl-4-phenyl-pyrrolidine 5f. Column chromatography: silica gel, AcOEt/ $/ \mathrm{NH}_{3}: 99 / 1$ then AcOEt/EtOH/ $/ \mathrm{NH}_{3}: 90 / 9 / 1$; colorless oil; Yield: $78 \%$; $\mathrm{R}_{\mathrm{f}}: 0.2$ $\left(\mathrm{AcOEt} / \mathrm{EtOH} / \mathrm{NH}_{3}: 90 / 9 / 1\right) ;[\alpha]_{\mathrm{D}}^{20}:+152\left(c \mathrm{0.8}, \mathrm{CHCl}_{3}\right) ;{ }^{1} \mathrm{H} \mathrm{NMR}\left(300 \mathrm{MHz}, \mathrm{CDCl}_{3}\right): \delta 7.04-$ $7.32(\mathrm{~m}, 10 \mathrm{H}), 4.00$ (A of ABq, $J=12.9 \mathrm{~Hz}, 1 \mathrm{H}), 3.26-3.43(\mathrm{~m}, 3 \mathrm{H}), 3.05$ (A' of A'B'X syst., $J$ $=8.7$ and $5.6 \mathrm{~Hz}, 1 \mathrm{H}$ ), 2.71 (A' of A'B'X syst., $J=10.8$ and $8.8 \mathrm{~Hz}, 1 \mathrm{H}$ ), 2.29 (B' of A'B'X syst., $J=8.7$ and $5.8 \mathrm{~Hz}, 1 \mathrm{H}), 1.48-1.70(\mathrm{~m}, 2 \mathrm{H}), 0.95(\mathrm{t}, J=7.3 \mathrm{~Hz}, 3 \mathrm{H}) ;{ }^{13} \mathrm{C} \mathrm{NMR}(75 \mathrm{MHz}$, $\left.\mathrm{CDCl}_{3}\right): \delta 139.4,138.6,128.8,128.7,128.3,128.2,126.9,126.4,74.8,59.4,58.5,55.3,47.7$, 26.1, 10.1; CIMS ( $\mathrm{NH}_{3}$ gas): 281.3, 264.3, 174.2; HRMS (CI, $\mathrm{NH}_{3}$ ) $m / z$ calcd for $\mathrm{C}_{19} \mathrm{H}_{25} \mathrm{~N}_{2}$ $[\mathrm{M}+\mathrm{H}]^{+} 281.202$ found 281.198 .

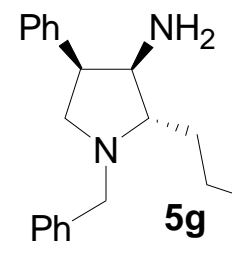

(2S,3R,4R)-3-Amino-1-benzyl-2-butyl-4-phenyl-pyrrolidine 5g. Column chromatography: silica gel, AcOEt/ $\mathrm{NH}_{3}$ : 99/1; yellow oil; Yield: $82 \%$; $\mathrm{R}_{\mathrm{f}}: 0.2$ (AcOEt/EtOH/NH$\left./ \mathrm{NH}_{3}: 97 / 2.8 / 0.2\right)$; $[\alpha]_{\mathrm{D}}^{20}:+112\left(c 1.0, \mathrm{CHCl}_{3}\right) ;{ }^{1} \mathrm{H}$ NMR $\left(300 \mathrm{MHz}, \mathrm{CDCl}_{3}\right): \delta 7.07-7.31(\mathrm{~m}, 10 \mathrm{H}), 3.96(\mathrm{~A}$ of $\mathrm{ABq}$, $J=12.9 \mathrm{~Hz}, 1 \mathrm{H}), 3.25-3.41(\mathrm{~m}, 3 \mathrm{H}), 3.03$ (A' of A'B'X syst., $J=8.7$ and $6.0 \mathrm{~Hz}, 1 \mathrm{H}), 2.69$ (B' of A'B'X syst., $J=11.2$ and $8.7 \mathrm{~Hz}, 1 \mathrm{H}), 2.32$ (app. q, $J=3.5 \mathrm{~Hz}, 1 \mathrm{H}), 1.23-1.64(\mathrm{~m}, 6 \mathrm{H}), 0.85$ $(\mathrm{t}, J=6.9 \mathrm{~Hz}, 3 \mathrm{H}) ;{ }^{13} \mathrm{C} \mathrm{NMR}\left(75 \mathrm{MHz}, \mathrm{CDCl}_{3}\right): \delta 139.5,138.7,128.9,128.7,128.4,128.2$, 126.9, 126.4, 73.7, 59.6, 59.2, 55.2, 47.7, 33.7, 28.3, 23.1, 14.2; CIMS ( $\mathrm{NH}_{3}$ gas): 309, 290, 251, 189; HRMS (CI, $\mathrm{NH}_{3}$ ) $m / z$ calcd for $\mathrm{C}_{21} \mathrm{H}_{29} \mathrm{~N}_{2}[\mathrm{M}+\mathrm{H}]^{+} 309.233$ found 309.231. 


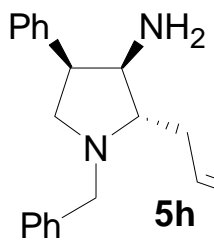

(2S,3R,4R)-2-Allyl-3-amino-1-benzyl-4-phenyl-pyrrolidin-3-ylamine

5h. Column chromatography: silica gel, AcOEt/MeOH/ $/ \mathrm{NH}_{3}$ : 99/4.5/0.5; colorless oil; Yield: $67 \%$; $\mathrm{R}_{\mathrm{f}}$ : 0.6 $\left(\mathrm{AcOEt} / \mathrm{MeOH} / \mathrm{NH}_{3}: 90 / 9 / 1\right) ;[\alpha]_{\mathrm{D}}^{20}:+64\left(c 1.25, \mathrm{CHCl}_{3}\right) ;{ }^{1} \mathrm{H} \mathrm{NMR}\left(300 \mathrm{MHz}, \mathrm{CDCl}_{3}\right): \delta 7.01-$ $7.24(\mathrm{~m}, 10 \mathrm{H}), 5.82-5.95(\mathrm{~m}, 1 \mathrm{H}), 5.08-5.10(\mathrm{~m}, 2 \mathrm{H}), 4.02(\mathrm{~A}$ of ABq, $J=12.9 \mathrm{~Hz}, 1 \mathrm{H}), 3.45$ (B of ABq, $J=12.9 \mathrm{~Hz}, 1 \mathrm{H}), 3.25-3.38(\mathrm{~m}, 2 \mathrm{H}), 3.05$ (A' of A'B'X syst., $J=8.5$ and $5.8 \mathrm{~Hz}, 1 \mathrm{H}$ ), 2.70 (A' of A'B'X syst., $J=9.6$ and $8.7 \mathrm{~Hz}, 1 \mathrm{H}), 2.43(\mathrm{~m}, 1 \mathrm{H}), 2.25-2.34(\mathrm{~m}, 2 \mathrm{H}) ;{ }^{13} \mathrm{C}$ NMR $(75$ $\left.\mathrm{MHz}, \mathrm{CDCl}_{3}\right): \delta 139.2,138.4,135.5,128.9,128.7,128.4,128.3,127.0,126.6,117.0,72.6,59.1$, 58.4, 55.5, 47.2, 37.8; CIMS ( $\mathrm{NH}_{3}$ gas): 293.3, 276.3, 186.2, 144.2, 115.1; HRMS (CI, $\left.\mathrm{NH}_{3}\right) \mathrm{m} / \mathrm{z}$ calcd for $\mathrm{C}_{20} \mathrm{H}_{24} \mathrm{~N}_{2}[\mathrm{M}]^{+} 292.194$ found 292.194.

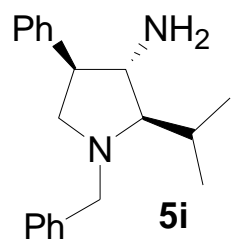

(2R,3S,4R)-3-Amino-1-benzyl-2-isopropyl-4-phenyl-pyrrolidine 5i. Column chromatography: silica gel, AcOEt/ $\mathrm{NH}_{3}$ : 99/1; colorless oil; Yield: $50 \%$; $\mathrm{R}_{\mathrm{f}}: 0.2\left(\mathrm{AcOEt} / \mathrm{EtOH} / \mathrm{NH}_{3}:\right.$ 97/2.8/0.2); $[\alpha]_{\mathrm{D}}^{20}:+42\left(c 0.5, \mathrm{CHCl}_{3}\right) ;{ }^{1} \mathrm{H} \mathrm{NMR}\left(300 \mathrm{MHz}, \mathrm{CDCl}_{3}\right): \delta 7.13-7.28(\mathrm{~m}, 10 \mathrm{H}) ; 3.75$ (A of ABq, $J$ $=12.7 \mathrm{~Hz}, 1 \mathrm{H}), 3.50-3.65(\mathrm{~m}, 4 \mathrm{H}), 2.97$ (app. $\mathrm{t}, J=5.8 \mathrm{~Hz}, 1 \mathrm{H}), 2.32(\mathrm{dd}, J=8.7$ and $3.1 \mathrm{~Hz}$, $1 \mathrm{H}), 1.42-1.56(\mathrm{~m}, 1 \mathrm{H}), 0.79(\mathrm{~d}, J=6.8 \mathrm{~Hz}, 3 \mathrm{H}), 0.43(\mathrm{~d}, J=6.5 \mathrm{~Hz}, 3 \mathrm{H}) ;{ }^{13} \mathrm{C} \mathrm{NMR}(75 \mathrm{MHz}$, $\left.\mathrm{CDCl}_{3}\right): \delta 140.9,137.4,129.0,128.9,128.6,128.5,127.5,126.8,73.8,61.5,58.8,58.3,36.6$, 29.0, 19.5, 19.2; CIMS ( $\mathrm{NH}_{3}$ gas): 295.3, 278.3; HRMS (CI, $\left.\mathrm{NH}_{3}\right) m / z$ calcd for $\mathrm{C}_{20} \mathrm{H}_{26} \mathrm{~N}_{2}[\mathrm{M}]^{+}$ 294.210 found 294.203 . 


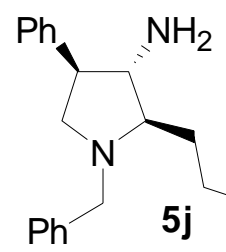

(2R,3S,4R)-3-Amino-1-benzyl-2-butyl-4-phenyl-pyrrolidine 5j. Column chromatography: silica gel, AcOEt/NH $:$ 99/1; colorless oil; Yield: $82 \%$; $\mathrm{R}_{\mathrm{f}}: 0.7$ (AcOEt/EtOH/NH$/$ : 90/9/1); $[\alpha]_{\mathrm{D}}^{20}:-46\left(c 1.0, \mathrm{CHCl}_{3}\right) ;{ }^{1} \mathrm{H} \mathrm{NMR}\left(300 \mathrm{MHz}, \mathrm{CDCl}_{3}\right): \delta 7.08-7.29(\mathrm{~m}, 10 \mathrm{H}), 4.05(\mathrm{~A}$ of $\mathrm{ABq}, J$ $=13.4 \mathrm{~Hz}, 1 \mathrm{H}), 3.11(\mathrm{~B}$ of ABq, $J=13.4 \mathrm{~Hz}, 1 \mathrm{H}), 3.06(\mathrm{dd}, J=7.7$ and $6.4 \mathrm{~Hz}, 1 \mathrm{H}), 2.87-2.90$ $(\mathrm{m}, 1 \mathrm{H}), 2.63-2.72(\mathrm{~m}, 2 \mathrm{H}), 2.24-2.30(\mathrm{~m}, 1 \mathrm{H}), 1.68-1.81(\mathrm{~m}, 6 \mathrm{H}), 0.85(\mathrm{t}, J=7.2 \mathrm{~Hz}, 3 \mathrm{H}) ;{ }^{13} \mathrm{C}$ NMR (75 MHz, $\left.\mathrm{CDCl}_{3}\right): \delta 144.8,139.7,128.6,128.5,128.2,127.4,126.7,126.2,72.4,64.9$, 59.4, 58.5, 52.8, 30.3, 26.7, 23.4, 14.2; CIMS ( $\mathrm{NH}_{3}$ gas): 309, 290, 251, 189, 176, 132; HRMS $\left(\mathrm{CI}, \mathrm{NH}_{3}\right) \mathrm{m} / z$ calcd for $\mathrm{C}_{21} \mathrm{H}_{29} \mathrm{~N}_{2}[\mathrm{M}+\mathrm{H}]^{+} 309.233$ found 309.230 .

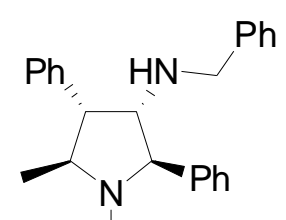

$5 k$

\section{(2R,3S,4R,5S)-3-Benzylamino-1,5-Dimethyl-2,4-diphenyl-pyrrolidine}

5k. Column chromatography: silica gel, $\mathrm{Et}_{2} \mathrm{O} / \mathrm{PE}: 70 / 30$; white solid; Yield: 88 \%; $\mathrm{R}_{\mathrm{f}}: 0.2\left(\mathrm{Et}_{2} \mathrm{O} / \mathrm{PE}: 70 / 30\right)$; Mp: $90{ }^{\circ} \mathrm{C} ;[\alpha]_{\mathrm{D}}^{20}:+17\left(c 0.8, \mathrm{CHCl}_{3}\right) ;{ }^{1} \mathrm{H}$ NMR $\left(300 \mathrm{MHz}, \mathrm{CDCl}_{3}\right): \delta$ 7.17-7.37 (m, 10H), 7.00$7.03(\mathrm{~m}, 3 \mathrm{H}), 6.64-6.67(\mathrm{~m}, 2 \mathrm{H}), 3.00-3.23(\mathrm{~m}, 5 \mathrm{H}), 2.71-2.80(\mathrm{~m}, 1 \mathrm{H}), 2.11(\mathrm{~s}, 3 \mathrm{H}), 1.07$ (d, 3H, $J=6.0 \mathrm{~Hz}) ;{ }^{13} \mathrm{C} \mathrm{NMR}\left(75 \mathrm{MHz}, \mathrm{CDCl}_{3}\right): \delta 140.2,138.9,128.3,128.2,128.1,128.0,127.8$, 127.2, 79.9, 67.0, 66.8, 56.1, 51.8, 39.4, 17.9; CIMS ( $\mathrm{NH}_{3}$ gas): 379.3, 357.3, 250.2, 239.2; HRMS (CI, $\mathrm{NH}_{3}$ ) $m / z$ calcd for $\mathrm{C}_{25} \mathrm{H}_{28} \mathrm{~N}_{2}[\mathrm{M}]^{+} 356.225$ found 356.231 . 


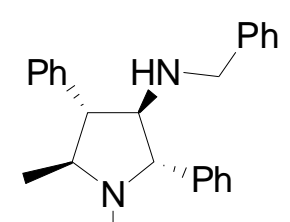

51

5l. Column chromatography: silica gel, $\mathrm{Et}_{2} \mathrm{O} / \mathrm{PE}$ : 70/30; colorless oil; Yield: 75 \%; $\mathrm{R}_{\mathrm{f}}: 0.2$ (Et $\left.2 \mathrm{O} / \mathrm{PE}: 70 / 30\right)$; $[\alpha]_{\mathrm{D}}^{20}:+33\left(c \quad 0.5, \mathrm{CHCl}_{3}\right) ;{ }^{1} \mathrm{H}$ NMR $\left(300 \mathrm{MHz}, \mathrm{CDCl}_{3}\right): \delta 7.13-7.34(\mathrm{~m}, 10 \mathrm{H}),, 7.01-7.05(\mathrm{~m}$, $3 \mathrm{H}), 6.76-6.79(\mathrm{~m}, 2 \mathrm{H}), 3.66(\mathrm{~d}, J=7.5 \mathrm{~Hz}, 1 \mathrm{H}), 3.21-3.38(\mathrm{~m}, 4 \mathrm{H}), 2.69(\mathrm{dd}, J=7.8$ and $5.7 \mathrm{~Hz}$, $1 \mathrm{H}), 2.04$ (s, 3H), 1.67 (br. s, $1 \mathrm{H}), 1.18$ (d, $J=6.5 \mathrm{~Hz}, 3 \mathrm{H}) ;{ }^{13} \mathrm{C} \mathrm{NMR}\left(75 \mathrm{MHz}, \mathrm{CDCl}_{3}\right.$ ): $\delta 144.2$, 142.0, 140.2, 128.6, 128.5, 128.3, 128.0, 127.8, 127.5, 74.4, 73.4, 65.8, 60.5, 52.3, 34.6, 15.9; CIMS $\left(\mathrm{NH}_{3}\right.$ gas): $379.3,357.3,250.2$; HRMS (CI, $\mathrm{NH}_{3}$ ) $\mathrm{m} / z$ calcd for $\mathrm{C}_{25} \mathrm{H}_{28} \mathrm{~N}_{2}[\mathrm{M}]^{+} 356.225$ found 356.234 . 


\section{Determination of configuration}

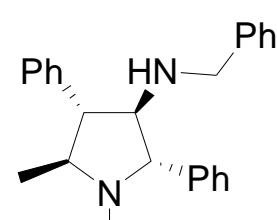

51

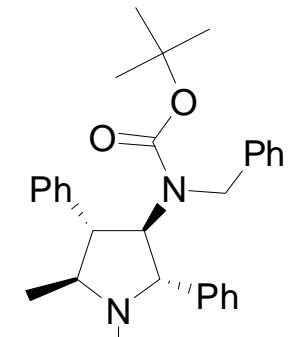

N-Boc-5I

(2S,3R,4R,5S)-3-(N-tert-Butoxycarbonyl-benzylamino)-1,5-Dimethyl-2,4-diphenyl-

pyrrolidine $N$-Boc-5l. To a solution of $5 \mathbf{l}(127 \mathrm{mg}, 0.35 \mathrm{mmol})$ in ethyl acetate $(8 \mathrm{~mL})$ was added di-tert-butyl dicarbonate $(85 \mathrm{mg}, 0.39 \mathrm{mmol})$. The resulting mixture was stirred at $\mathrm{rt}$ for 2 hours, concentrated and purified by column chromatography (silica gel, AcOEt/PE: 25/75) to yield the desired carbamate as colorless crystals $(127 \mathrm{mg}, 0.28 \mathrm{mmol}, 79 \%)$. $\mathrm{R}_{\mathrm{f}}: 0.75$ (AcOEt/PE/NH $\left.\mathrm{NH}_{3}: 25 / 74 / 1\right)$; Mp: $112{ }^{\circ} \mathrm{C} ;[\alpha]_{\mathrm{D}}^{20}:+19\left(c\right.$ 0.5, $\left.\mathrm{CHCl}_{3}\right) ;{ }^{1} \mathrm{H}$ NMR (300 MHz, DMSO$d_{6}+1$ drop $\left.\mathrm{D}_{2} \mathrm{O}, 343 \mathrm{~K}\right): \delta 7.30-7.33(\mathrm{~m}, 15 \mathrm{H}), 4.70$ (br. s, $\left.1 \mathrm{H}\right), 4.38(\mathrm{~s}, 2 \mathrm{H}), 3.98(\mathrm{~d}, J=8.5 \mathrm{~Hz}$, 1H), 3.33 (q, $J=6.2 \mathrm{~Hz}, 1 \mathrm{H}), 2.92-2.98(\mathrm{~m}, 1 \mathrm{H}), 2.05(\mathrm{~s}, 3 \mathrm{H}), 1.08(\mathrm{~d}, J=6.3 \mathrm{~Hz}, 3 \mathrm{H}), 1.06$ (s, $9 \mathrm{H}) ;{ }^{13} \mathrm{C}$ NMR (75 MHz, DMSO- $\left.d_{6}, 333 \mathrm{~K}\right): \delta 154.6,142.0,140.7,139.2,127.9,127.7,127.6$, $127.5,127.2,126.8,126.5,126.0,125.9,78.3,71.3,63.8,53.7,47.3,33.8,27.2,15.6$; CIMS $\left(\mathrm{NH}_{3}\right.$ gas): 401.3, 379.3, 250.2; HRMS (CI, $\mathrm{NH}_{3}$ ) $m / z$ calcd for $\mathrm{C}_{30} \mathrm{H}_{36} \mathrm{~N}_{2} \mathrm{O}_{2}[\mathrm{M}]^{+} 456.278$ found 456.276 .

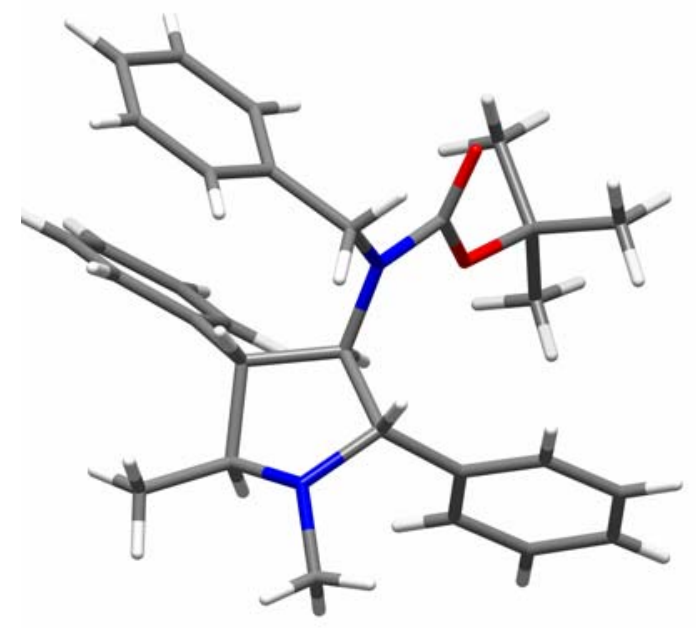




\section{Synthesis of (-)-Absouline.}

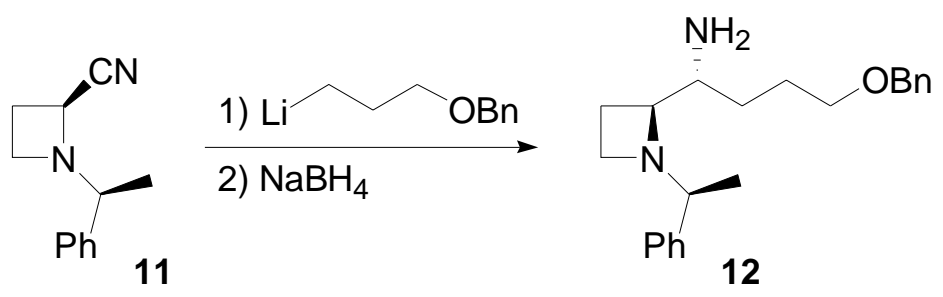

[1(1S),2S,2(1R)]-2-(1-Amino-4-benzyloxy-butyl)-1-(1-phenyl-ethyl)-azetidine $12 . \quad$ To a solution of 1-benzyloxy-3-bromopropane $(5.16 \mathrm{~g}, 22.5 \mathrm{mmol})$ in dry ether $(50 \mathrm{~mL})$ was slowly added at $-78{ }^{\circ} \mathrm{C}$ tert-butyllithium $(1.5 \mathrm{M}$ solution in pentane, $31.0 \mathrm{~mL}, 46.5 \mathrm{mmol})$ under argon. The reaction mixture was slowly warmed to $\mathrm{rt}$ and then added dropwise to a solution of cyanoazetidine $\mathbf{1 1}^{3}(1.0 \mathrm{~g}, 5.5 \mathrm{mmol})$ in dry toluene $(50 \mathrm{~mL})$ at $0{ }^{\circ} \mathrm{C}$. The reaction mixture was then stirred for $2 \mathrm{~h}$ at $0^{\circ} \mathrm{C}, 30 \mathrm{~min}$ at $\mathrm{rt}$, cooled to $0{ }^{\circ} \mathrm{C}$ and carefully quenched with $\mathrm{MeOH}$. Sodium borohydride ( $853 \mathrm{mg}, 22.5 \mathrm{mmol}$ ) was then added and the reaction was warmed to $\mathrm{rt}$ and stirred overnight. Saturated aqueous ammonium chloride solution was finally added to quench the reaction mixture which was then concentrated under reduced pressure. The resulting slurry was diluted in ethyl acetate and the solution was basified with aqueous hydroxide sodium $(50 \mathrm{~mL}$ of a $2 \mathrm{M}$ solution). Layers were separated, the aqueous layer was extracted with dichloromethane $(3 \mathrm{x}$ $100 \mathrm{~mL}$ ), and the combined organic layers were dried over $\mathrm{MgSO}_{4}$. Evaporation of the solvent under reduced pressure and flash column chromatography of the residue (silica gel, AcOEt followed by $\mathrm{AcOEt} / \mathrm{EtOH} / \mathrm{NH}_{3}:$ 95/4/1) afforded compound 12 as a colorless oil (1.2 g, 3.5 mmol, 64 \%). Rf: 0.2 (AcOEt/EtOH/NH $\left./ \mathrm{NH}_{3}: 90 / 9 / 1\right) ;[\alpha]_{\mathrm{D}}^{20}:-47$ (c 1.6, $\left.\mathrm{CHCl}_{3}\right) ;{ }^{1} \mathrm{H} \mathrm{NMR}(300 \mathrm{MHz}$, $\left.\mathrm{CDCl}_{3}\right): \delta 7.12-7.27(\mathrm{~m}, 10 \mathrm{H}), 4.49(\mathrm{~s}, 2 \mathrm{H}), 3.21-3.48(\mathrm{~m}, 4 \mathrm{H}), 2.83-2.91(\mathrm{~m}, 2 \mathrm{H}), 2.48-2.56(\mathrm{~m}$, $1 \mathrm{H}), 1.86-2.08(\mathrm{~m}, 3 \mathrm{H}), 1.52-1.78(\mathrm{~m}, 3 \mathrm{H}), 1.14-1.31(\mathrm{~m}, 2 \mathrm{H}), 1.09-1.12(\mathrm{~d}, J=6.5 \mathrm{~Hz}, 3 \mathrm{H}) ;{ }^{13} \mathrm{C}$ NMR (75 MHz, $\left.\mathrm{CDCl}_{3}\right): \delta 144.4,138.6,128.4,128.3,127.7,127.6,127.2,126.9,73.0,70.5$, 68.9, 67.8, 52.6, 49.6, 29.7, 26.8 22.6, 14.5; CIMS ( $\mathrm{NH}_{3}$ gas): 339, 320, 122, 106; HRMS (CI, $\mathrm{NH}_{3}$ ) $m / z$ calcd for $\mathrm{C}_{22} \mathrm{H}_{31} \mathrm{~N}_{2} \mathrm{O}[\mathrm{M}+\mathrm{H}]^{+} 339.244$ found 339.243 .

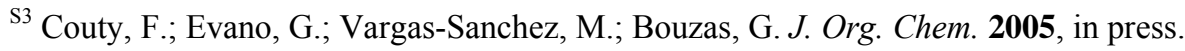




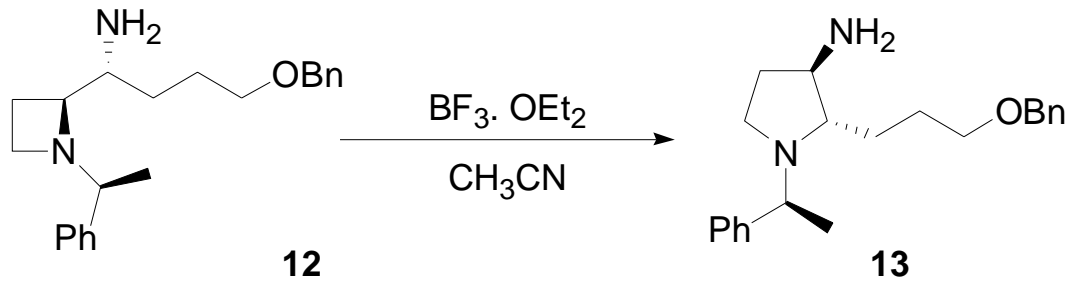

[1(1S),2S,3R]-3-Amino-2-(3-benzyloxy-propyl)-1-(1-phenyl-ethyl)-pyrrolidine 13. To a stirred solution of the $12(500 \mathrm{mg}, 1.47 \mathrm{mmol})$ in acetonitrile $(20 \mathrm{~mL})$ was added $\mathrm{BF}_{3} \cdot \mathrm{OEt}_{2}(190$ $\mu \mathrm{L}, 1.48 \mathrm{mmol}$ ) at room temperature. The resulting mixture was refluxed for $12 \mathrm{~h}$, quenched by addition of an aqueous saturated solution of sodium bicarbonate $(20 \mathrm{~mL})$, and stirred at room temperature for $5 \mathrm{~min}$. The aqueous layer was extracted with diethyl ether ( $3 \times 25 \mathrm{~mL}$ ), and the combined organic layers were dried over $\mathrm{MgSO}_{4}$, filtered, and concentred. The residue was purified by flash column chromatography (silica gel, AcOEt/NH $\mathrm{NH}_{3}$ :99/1) which provided pure compound 13 as a colorless oil (400 mg, 80\%). $\mathrm{R}_{\mathrm{f}}: 0.1\left(\mathrm{AcOEt} / \mathrm{EtOH} / \mathrm{NH}_{3}: 90 / 9 / 1\right) ;[\alpha]_{\mathrm{D}}^{20}:-3(c$ 0.5, $\left.\mathrm{CHCl}_{3}\right) ;{ }^{1} \mathrm{H} \mathrm{NMR}\left(300 \mathrm{MHz}, \mathrm{CDCl}_{3}\right): \delta 7.10-7.30(\mathrm{~m}, 10 \mathrm{H}), 4.37$ (s, 2H), 3.70 (q, $J=6.5$ $\mathrm{Hz}, 1 \mathrm{H}), 3.28(\mathrm{t}, J=6.4 \mathrm{~Hz}, 2 \mathrm{H}), 3.10(\mathrm{dt}, J=6.6$ and $2.4 \mathrm{~Hz}, 1 \mathrm{H}), 2.63(\mathrm{dd}, J=8.5$ and $5.2 \mathrm{~Hz}$, 2H), 2.49 (dt, $J=9.0$ and $2.9 \mathrm{~Hz}, 1 \mathrm{H}), 1.85-1.93(\mathrm{~m}, 2 \mathrm{H}), 1.28(\mathrm{~d}, J=6.5 \mathrm{~Hz}, 3 \mathrm{H}), 1.08-1.64(\mathrm{~m}$, $4 \mathrm{H}) ;{ }^{13} \mathrm{C} \mathrm{NMR}\left(75 \mathrm{MHz}, \mathrm{CDCl}_{3}\right): \delta 145.1,138.6,128.4,128.3,128.1,127.6,127.5,126.8,72.9$, 70.6, 70.4, 60.2, 55.7, 47.2, 33.3, 28.3, 26.3, 17.8; CIMS ( $\mathrm{NH}_{3}$ gas): 339, 320, 282; HRMS (CI, $\mathrm{NH}_{3}$ ) $m / z$ calcd for $\mathrm{C}_{22} \mathrm{H}_{31} \mathrm{~N}_{2} \mathrm{O}[\mathrm{M}+\mathrm{H}]^{+} 339.244$ found 339.240 .

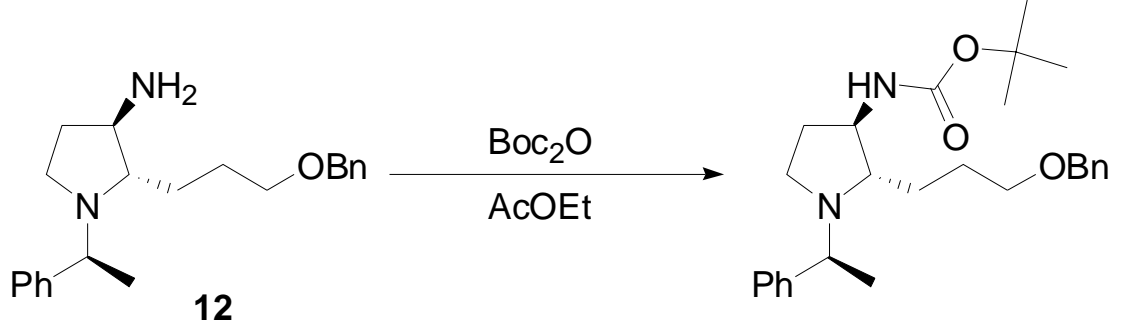

\section{[1(1S),2S,3R]-2-(3-Benzyloxy-propyl)-3-(tert-butoxycarbonylamino)-1-(1-phenyl-ethyl)-}

pyrrolidine. To a solution of $12(484 \mathrm{mg}, 1.43 \mathrm{mmol})$ in ethyl acetate $(30 \mathrm{~mL})$ was added di-tertbutyl dicarbonate $(343 \mathrm{mg}, 1.57 \mathrm{mmol}$ ). The resulting mixture was stirred at $\mathrm{rt}$ for $30 \mathrm{~min}$, concentrated and purified by column chromatography (silica gel, AcOEt/PE 70/30) to yield the desired carbamate as a colorless oil (626 mg, quant.). $\mathrm{R}_{\mathrm{f}}$ : 0.1 (AcOEt/PE: 70/30); [ $\left.\alpha\right]_{\mathrm{D}}^{20}:-2(c 1.1$, 
$\left.\mathrm{CHCl}_{3}\right) ;{ }^{1} \mathrm{H}$ NMR $\left(300 \mathrm{MHz}, \mathrm{DMSO}-d_{6}, 318 \mathrm{~K}\right): \delta$ 7.15-7.34 (m, 10H), 6.72 (br. s, $\left.1 \mathrm{H}\right), 4.39$ (s, $2 \mathrm{H}), 3.78-3.84(\mathrm{~m}, 1 \mathrm{H}), 3.60-3.64(\mathrm{~m}, 1 \mathrm{H}), 3.31(\mathrm{~m}, 2 \mathrm{H}), 3.20(\mathrm{~m}, 1 \mathrm{H}), 2.55-2.66(\mathrm{~m}, 2 \mathrm{H}), 1.76-$ $1.89(\mathrm{~m}, 1 \mathrm{H}), 1.32-1.66(\mathrm{~m}, 5 \mathrm{H}), 1.38(\mathrm{~s}, 9 \mathrm{H}), 1.26(\mathrm{~d}, J=6.6 \mathrm{~Hz}, 3 \mathrm{H}) ;{ }^{13} \mathrm{C} \mathrm{NMR}(75 \mathrm{MHz}$, $\left.\mathrm{CDCl}_{3}\right): \delta 155.2,146.7,145.0,128.3,128.2,127.6,127.5,126.9,85.2,72.9,70.4,67.0,59.2$, 54.4, 46.9, 30.9, 29.7, 28.5, 26.0, 18.7; CIMS ( $\mathrm{NH}_{3}$ gas): 439, 333, 289, 233; HRMS (CI, $\mathrm{NH}_{3}$ ) $m / z$ calcd for $\mathrm{C}_{27} \mathrm{H}_{39} \mathrm{~N}_{2} \mathrm{O}_{3}[\mathrm{M}+\mathrm{H}]^{+} 439.296$ found 439.292 .

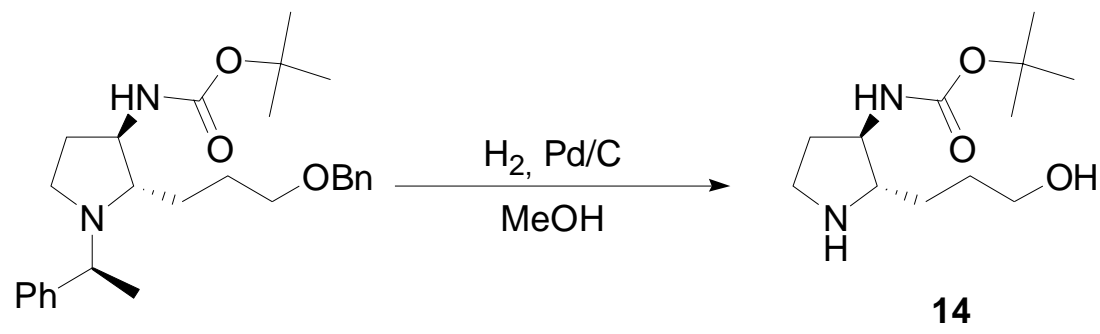

[1(1S),2S,3R]-3-(tert-Butoxycarbonylamino)-2-(3-hydroxypropyl)-pyrrolidine 14. A solution of [1(1S),2S,3R]-2-(3-benzyloxy-propyl)-3-(tert-butoxycarbonylamino)-1-(1-phenyl-ethyl)pyrrolidine $(352 \mathrm{mg}, 0.80 \mathrm{mmol})$ in $\mathrm{MeOH}(20 \mathrm{~mL})$ was treated with palladium on carbon $(10 \%$ wt., $352 \mathrm{mg}$ ) and stirred under an atmosphere of hydrogen for $12 \mathrm{~h}$. The mixture was filtered over a plug of Celite ${ }^{\circledR}$ which was rinsed with methanol and concentrated to yield $\mathbf{1 4}$ as a white solid which was used in the next step without further purification (182 mg, $0.74 \mathrm{mmol}, 93 \%)$. $\mathrm{R}_{\mathrm{f}}: 0.1$ $\left(\mathrm{CH}_{2} \mathrm{Cl}_{2} / \mathrm{MeOH} / \mathrm{NH}_{3}: 70 / 28 / 2\right) ; \mathrm{Mp}: 82{ }^{\circ} \mathrm{C} ;[\alpha]_{\mathrm{D}}^{20}$ : -7 (c $\left.1.0, \mathrm{CHCl}_{3}\right) ;{ }^{1} \mathrm{H}$ NMR $(300 \mathrm{MHz}$, DMSO- $\left.d_{6}, 345 \mathrm{~K}\right): \delta 6.48$ (br. s, $\left.1 \mathrm{H}\right), 3.48-3.56(\mathrm{~m}, 1 \mathrm{H}), 3.39-3.47(\mathrm{~m}, 2 \mathrm{H}), 2.83-2.92(\mathrm{~m}, 2 \mathrm{H})$, 2.70-2.79 (m, 1H), 1.90-2.00 (m, 1H), $1.40(\mathrm{~s}, 9 \mathrm{H}), 1.27-1.74(\mathrm{~m}, 5 \mathrm{H}) ;{ }^{13} \mathrm{C}$ NMR $(75 \mathrm{MHz}$, DMSO- $\left.d_{6}, 295 \mathrm{~K}\right): \delta 155.2,78.1,63.6,60.9,55.9,43.6,32.2,30.1,29.9,28.2 ; \mathrm{CIMS}\left(\mathrm{NH}_{3}\right.$ gas $)$ : 267.2, 245.2, 189.1; HRMS (CI, $\mathrm{NH}_{3}$ ) $m / z$ calcd for $\mathrm{C}_{12} \mathrm{H}_{25} \mathrm{~N}_{2} \mathrm{O}_{3}[\mathrm{M}+\mathrm{H}]^{+} 245.186$ found 245.185. 


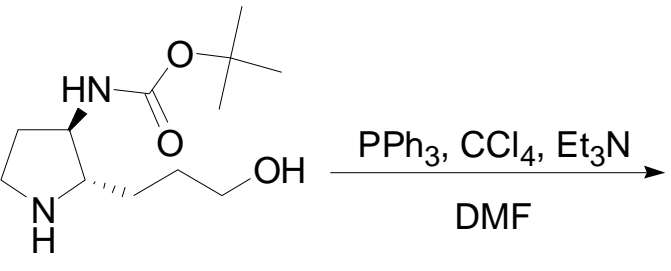

14

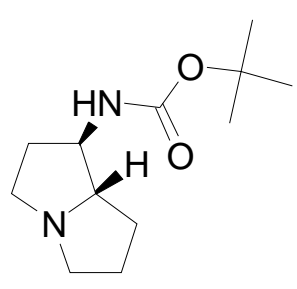

15

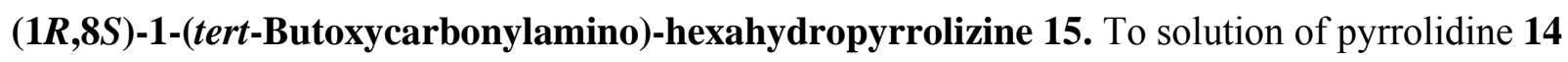
$(65 \mathrm{mg}, 0.26 \mathrm{mmol})$ in dry DMF $(2 \mathrm{~mL})$ were successively added triphenylphosphine (139 mg, $0.53 \mathrm{mmol}), \mathrm{CCl}_{4}(51 \mu \mathrm{L}, 0.53 \mathrm{mmol})$ and $\mathrm{Et}_{3} \mathrm{~N}(74 \mu \mathrm{L}, 0.53 \mathrm{mmol})$. The reaction mixture was stirred in the dark at room temperature for $72 \mathrm{~h}$ and quenched with the $\mathrm{MeOH}(1.5 \mathrm{~mL})$. The resulting mixture was stirred for $30 \mathrm{~min}$ and solvents were evaporated. The residue was carefully basified to $\mathrm{pH} 9$ with a $1 \mathrm{M}$ solution of potassium hydroxide $(1 \mathrm{~mL})$ and stirred for an additional 20 min. After removal of water under reduced pressure, the residue was purified by column chromatography (basic alumina, $\mathrm{CH}_{2} \mathrm{Cl}_{2} / \mathrm{EtOH}$ : 99/1), affording 15 as a pale yellow solid (45 $\mathrm{mg}$, 0.20 mmol, 76 \%). $\mathrm{R}_{\mathrm{f}}: 0.1\left(\mathrm{CH}_{2} \mathrm{Cl}_{2} / \mathrm{MeOH} 98: 2\right)$; $\mathrm{Mp}: 115{ }^{\circ} \mathrm{C} ;[\alpha]_{\mathrm{D}}^{20}:-10\left(c \quad 0.7, \mathrm{CHCl}_{3}\right) ;{ }^{1} \mathrm{H}$ NMR (300 MHz, $\left.\mathrm{CDCl}_{3}\right): \delta 4.70$ (br. s, $\left.1 \mathrm{H}\right), 3.71-3.80(\mathrm{~m}, 1 \mathrm{H}), 3.12-3.18(\mathrm{~m}, 2 \mathrm{H}), 2.96(\mathrm{dt}, J=$ 10.4 and $6.0 \mathrm{~Hz}, 1 \mathrm{H}), 2.52-2.62(\mathrm{~m}, 2 \mathrm{H}), 2.12-2.22(\mathrm{~m}, 1 \mathrm{H}), 1.87-2.01(\mathrm{~m}, 1 \mathrm{H}), 1.57-1.87(\mathrm{~m}$, $4 \mathrm{H}), 1.45(\mathrm{~s}, 9 \mathrm{H}) ;{ }^{13} \mathrm{C} \mathrm{NMR}\left(75 \mathrm{MHz}, \mathrm{CDCl}_{3}\right): \delta 155.6,76.2,70.8,56.8,55.3,53.2,33.2,30.7$, 28.4, 25.4; CIMS ( $\mathrm{NH}_{3}$ gas): 227.1, 219.0, 171.0; HRMS (CI, $\left.\mathrm{NH}_{3}\right) \mathrm{m} / z$ calcd for $\mathrm{C}_{12} \mathrm{H}_{23} \mathrm{~N}_{2} \mathrm{O}_{2}$ $[\mathrm{M}+\mathrm{H}]^{+} 227.176$ found 227.175 . 


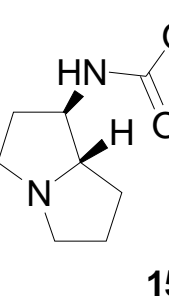

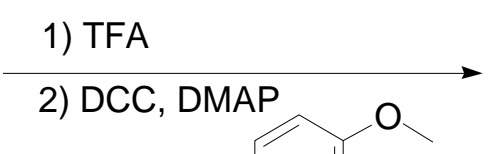

$\mathrm{HO}$

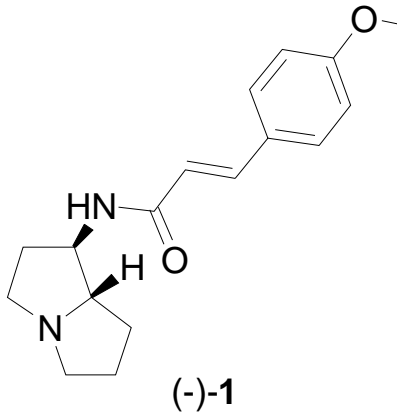

$(-)-1$

(-)-Absouline (-)-1. To a solution of $15(37 \mathrm{mg}, 0.16 \mathrm{mmol})$ in dichloromethane $(4 \mathrm{~mL})$ was added trifluoroacetic acid $(1.5 \mathrm{~mL})$ at $0^{\circ} \mathrm{C}$. The mixture was stirred for 20 min at $0{ }^{\circ} \mathrm{C}$, slowly warmed to rt, stirred for an additional $20 \mathrm{~min}$ and concentrated in vacuum. Traces of TFA were removed by drying the product overnight on high vacuum. The obtained solid was dissolved in dichloromethane $(4 \mathrm{~mL})$ and DMAP $(10 \mathrm{mg}, 0.08 \mathrm{mmol})$ and trans-4-methoxycinnamic acid (34 $\mathrm{mg}, 0.19 \mathrm{mmol})$ were added. The mixture was cooled to $0{ }^{\circ} \mathrm{C}$ before adding DCC $(66 \mathrm{mg}, 0.32$ mmol) in one portion. The resulting mixture was stirred at $0{ }^{\circ} \mathrm{C}$ for $15 \mathrm{~min}$, slowly warmed to $\mathrm{rt}$ and stirred for $30 \mathrm{~min}$. Crude mixture was filtered and diluted with saturated $\mathrm{NaHCO}_{3}$ aqueous solution $(1 \mathrm{~mL})$. The aqueous layer was extracted with $\mathrm{CH}_{2} \mathrm{Cl}_{2}$ and the combined organic layers were dried over $\mathrm{MgSO}_{4}$, filtered, and concentrated. The residue was purified by column chromatography (basic alumina, $\mathrm{CH}_{2} \mathrm{Cl}_{2}$ ), affording synthetic absouline (-)-1 as colorless crystals (25 mg, 0.09 mmol, 55 \%). $\mathrm{R}_{\mathrm{f}}$ : 0.3 (basic alumina, $\mathrm{CH}_{2} \mathrm{Cl}_{2} / \mathrm{MeOH}: 97 / 3$ ); Mp: $165^{\circ} \mathrm{C}$ \{Lit.: Mp: $186{ }^{\circ} \mathrm{C}$ (acetone) $\left.)^{\mathrm{S} 4}\right\} ;[\alpha]_{\mathrm{D}}^{20}:-37\left(c 1.8, \mathrm{CHCl}_{3}\right)$, \{Lit.: synthetic (-)-absouline: $[\alpha]_{\mathrm{D}}^{20}:-34(c 0.91$, $\left.\left.\mathrm{CHCl}_{3}\right)^{\mathrm{S} 5}\right\} ; \quad[\alpha]_{\mathrm{D}}^{20}:-51(c 0.4, \mathrm{EtOH})\left\{\right.$ Lit.: natural $(+)$-absouline: $[\alpha]_{\mathrm{D}}^{20}:+56(c 1, \mathrm{EtOH}),{ }^{\mathrm{S} 4}$ synthetic (+)-absouline: $\left.[\alpha]_{\mathrm{D}}^{20}:+26\left(c 1.05, \mathrm{CHCl}_{3}\right)^{\mathrm{S} 5}\right\}$; IR $\left(\mathrm{NaCl}\right.$, film) $v_{\max }: 3418,3257,2932$, 1660, $1603 \mathrm{~cm}^{-1} ;{ }^{1} \mathrm{H}$ NMR (300 MHz, $\left.\mathrm{CDCl}_{3}\right): \delta 7.57$ (d, $\left.J=15.6 \mathrm{~Hz}, 1 \mathrm{H}\right), 7.44(\mathrm{~d}, J=8.6 \mathrm{~Hz}$, 2H), 6.87 (d, $J=8.7 \mathrm{~Hz}, 2 \mathrm{H}), 6.29$ (d, $J=15.6 \mathrm{~Hz}, 1 \mathrm{H}), 6.28$ (obsc. d, 1H), 4.25 (app. quint., $J=$ $6.7 \mathrm{~Hz}, 1 \mathrm{H}), 3.82(\mathrm{~s}, 3 \mathrm{H}), 3.20-3.34(\mathrm{~m}, 1 \mathrm{H}), 2.98-3.06(\mathrm{~m}, 1 \mathrm{H}), 2.59-2.69(\mathrm{~m}, 2 \mathrm{H}), 2.20-2.31(\mathrm{~m}$, $1 \mathrm{H}), 1.97-2.17(\mathrm{~m}, 1 \mathrm{H}), 1.56-1.91(\mathrm{~m}, 4 \mathrm{H}) ;{ }^{13} \mathrm{C} \mathrm{NMR}\left(75 \mathrm{MHz}, \mathrm{CDCl}_{3}\right): \delta 166.2,160.9,140.7$, 129.4, 127.6, 118.2, 114.2, 71.0, 55.4, 55.4, 55.2, 53.4, 33.0, 30.7, 25.4; CIMS ( $\mathrm{NH}_{3}$ gas): 287.2; HRMS (CI, $\left.\mathrm{NH}_{3}\right) m / z$ calcd for $\mathrm{C}_{12} \mathrm{H}_{23} \mathrm{~N}_{2} \mathrm{O}_{2}[\mathrm{M}+\mathrm{H}]^{+} 287.1760$ found 287.1711 .

\footnotetext{
${ }^{S 4}$ Ikhiri, K.; Ahond, A.; Poupat, C.; Potier, P.; Pusset, J.; Sévenet, T. J. Nat. Prod. 1987, 50, 626-630.

${ }^{S}$ Christine, C.; Ikhiri, K.; Ahond, A.; Al Mourabit, A.; Poupat, C.; Potier, P. Tetrahedron 2000, 56, 1837-1850.
} 


\section{Absouline Spectral Data Compared to Reported Data}

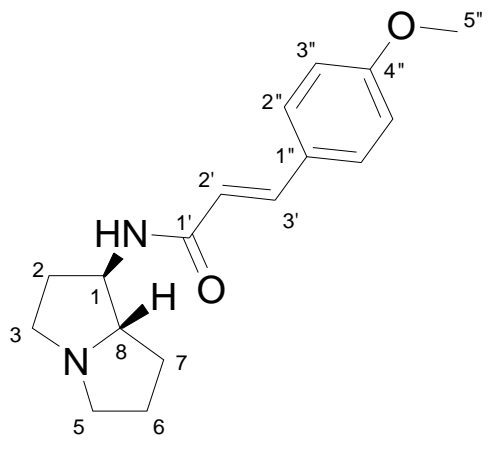

${ }^{1}$ H NMR data

\begin{tabular}{|c|c|c|c|c|c|c|c|c|c|c|c|}
\hline \multicolumn{12}{|c|}{${ }^{1}$ H NMR data } \\
\hline \multicolumn{4}{|c|}{ Our synthesis } & \multicolumn{4}{|c|}{ Ikhiri, Ahond and Poupat (400 MHz) } & \multicolumn{4}{|c|}{ Poupat (300 MHz) ${ }^{\text {S7 }}$} \\
\hline Attribution & $\delta$ & Int. & Mult. & Attribution & $\delta$ & Int. & Mult. & Attribution & $\delta$ & Int. & Mult. \\
\hline $\mathrm{H}_{3}$ & 7.57 & $1 \mathrm{H}$ & $\begin{array}{c}\mathrm{d}, J=15.6 \\
\mathrm{~Hz}\end{array}$ & $\mathrm{H}_{2}$, or $\mathrm{H}_{3}$ & 7.55 & $1 \mathrm{H}$ & $\mathrm{d}, J=16 \mathrm{~Hz}$ & $\mathrm{H}_{3}$ & 7.59 & $1 \mathrm{H}$ & $\begin{array}{c}\mathrm{d}, J=16.0 \\
\mathrm{~Hz}\end{array}$ \\
\hline $\mathrm{H}_{2}$, & 7.44 & $2 \mathrm{H}$ & $\mathrm{d}, J=8.6 \mathrm{~Hz}$ & $\mathrm{H}_{2}$, or $\mathrm{H}_{3^{\prime \prime}}$ & 7.41 & $2 \mathrm{H}$ & $\mathrm{d}, J=9 \mathrm{~Hz}$ & $\mathrm{H}_{2}$, & 7.45 & $2 \mathrm{H}$ & $\mathrm{d}, J=9.0 \mathrm{~Hz}$ \\
\hline & & & & & & & & $\mathrm{NH}$ & 7,30 & $1 \mathrm{H}$ & br. d \\
\hline $\mathrm{H}_{3}$ & 6.87 & $2 \mathrm{H}$ & $\mathrm{d}, J=8.7 \mathrm{~Hz}$ & $\mathrm{H}_{2}^{\prime \prime}$ or $\mathrm{H}_{3^{\prime \prime}}$ & 6.86 & $2 \mathrm{H}$ & $\mathrm{d}, J=9 \mathrm{~Hz}$ & $\mathrm{H}_{3}$ & 6.87 & $2 \mathrm{H}$ & $\mathrm{d}, J=9.0 \mathrm{~Hz}$ \\
\hline $\mathrm{H}_{2}$ & 6.29 & $1 \mathrm{H}$ & $\begin{array}{c}\mathrm{d}, J=15.6 \\
\mathrm{~Hz}\end{array}$ & $\mathrm{H}_{2}$, or $\mathrm{H}_{3}$ & 6.23 & $1 \mathrm{H}$ & $\mathrm{d}, J=16 \mathrm{~Hz}$ & $\mathrm{H}_{2}$ & 6.40 & $1 \mathrm{H}$ & $\begin{array}{c}\mathrm{d}, J=16.0 \\
\mathrm{~Hz}\end{array}$ \\
\hline $\mathrm{NH}$ & 6.28 & $1 \mathrm{H}$ & obsc. d & $\mathrm{NH}$ & 5.84 & $1 \mathrm{H}$ & $\begin{array}{l}\text { br. d, } J= \\
10.5 \mathrm{~Hz}\end{array}$ & & & & \\
\hline
\end{tabular}

${ }^{\mathrm{S} 6}$ Comparison data from isolation of absoulin, as disclosed by Ikhiri, Ahond and Poupat : Ikhiri, K.; Ahond, A.; Poupat, C.; Potier, P.; Pusset, J.; Sevene, T. J. Nat. Prod. 1987, 50, 626-630.

${ }^{\mathrm{S} 7}$ Comparison data from synthesis of absoulin, as disclosed by Poupat: Christine, C.; Ikhiri, K.; Ahond, A.; Al Mourabit, A.; Poupat, C.; Potier, P. Tetrahedron 2000, 56, 18371850. 


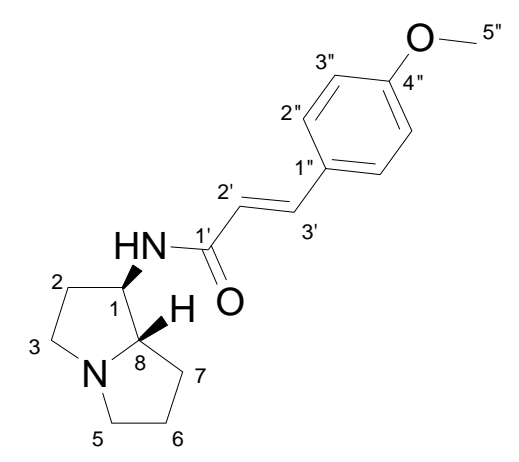

\begin{tabular}{|c|c|c|c|c|c|c|c|c|c|c|c|}
\hline $\mathrm{H}_{1}$ & 4.25 & $1 \mathrm{H}$ & $\begin{array}{c}\text { app. quint., } J \\
=6.7 \mathrm{~Hz}\end{array}$ & $\mathrm{H}_{1}$ & 4.22 & $1 \mathrm{H}$ & $\mathrm{m}$ & $\mathrm{H}_{1}$ & 4.33 & $1 \mathrm{H}$ & $\mathrm{m}$ \\
\hline $\mathrm{H}_{5}$ & 3.82 & $3 \mathrm{H}$ & $\mathrm{s}$ & $\mathrm{H}_{5}$ & 3.80 & $3 \mathrm{H}$ & $\mathrm{s}$ & $\mathrm{H}_{5}$ & 3.80 & $3 \mathrm{H}$ & $\mathrm{s}$ \\
\hline \multirow{2}{*}{$\begin{array}{c}\mathrm{H}_{8} \\
1 \mathrm{H}_{3} \\
\end{array}$} & $3.20-$ & \multirow{2}{*}{$2 \mathrm{H}$} & \multirow{2}{*}{$\mathrm{m}$} & $\mathrm{H}_{8}$ & 3.24 & $1 \mathrm{H}$ & $\mathrm{m}$ & $\mathrm{H}_{8}$ & 3.58 & $1 \mathrm{H}$ & $\mathrm{m}$ \\
\hline & 3.24 & & & $1 \mathrm{H}_{3}$ & 3.20 & $1 \mathrm{H}$ & $\mathrm{m}$ & $1 \mathrm{H}_{3}$ & 3.45 & $1 \mathrm{H}$ & $\mathrm{m}$ \\
\hline $1 \mathrm{H}_{5}$ & $\begin{array}{l}2.98- \\
3.06\end{array}$ & $1 \mathrm{H}$ & $\mathrm{m}$ & $1 \mathrm{H}_{5}$ & 2.98 & $1 \mathrm{H}$ & $\mathrm{m}$ & $1 \mathrm{H}_{5}$ & 3.19 & $1 \mathrm{H}$ & $\mathrm{m}$ \\
\hline \multirow{2}{*}{$\begin{array}{l}1 \mathrm{H}_{3}, \\
1 \mathrm{H}_{5} \\
\end{array}$} & $2.59-$ & \multirow{2}{*}{$2 \mathrm{H}$} & \multirow{2}{*}{$\mathrm{m}$} & $1 \mathrm{H}_{5}$ & 2.62 & $1 \mathrm{H}$ & $\mathrm{m}$ & \multirow{2}{*}{$\begin{array}{l}1 \mathrm{H}_{3} \\
1 \mathrm{H}_{5}\end{array}$} & \multirow{2}{*}{2.72} & \multirow{2}{*}{$2 \mathrm{H}$} & \multirow{2}{*}{$\mathrm{m}$} \\
\hline & 2.69 & & & $1 \mathrm{H}_{3}$ & 2.60 & $1 \mathrm{H}$ & $\mathrm{m}$ & & & & \\
\hline $1 \mathrm{H}_{2}$ & $\begin{array}{c}2.20- \\
2.31\end{array}$ & $1 \mathrm{H}$ & $\mathrm{m}$ & $1 \mathrm{H}_{2}$ & 2.25 & $1 \mathrm{H}$ & $\mathrm{m}$ & $1 \mathrm{H}_{2}$ & 2.28 & $1 \mathrm{H}$ & $\mathrm{m}$ \\
\hline $1 \mathrm{H}_{7}$ & $\begin{array}{l}1.97- \\
2.17 \\
\end{array}$ & $1 \mathrm{H}$ & $\mathrm{m}$ & $1 \mathrm{H}_{7}$ & 1.98 & $1 \mathrm{H}$ & $\mathrm{m}$ & $1 \mathrm{H}_{7}$ & 2.08 & $1 \mathrm{H}$ & $\mathrm{m}$ \\
\hline \multirow{4}{*}{$\begin{array}{c}1 \mathrm{H}_{2} \\
\mathrm{H}_{6} \\
1 \mathrm{H}_{7}\end{array}$} & \multirow{4}{*}{$\begin{array}{c}1.56- \\
1.91\end{array}$} & \multirow{4}{*}{$4 \mathrm{H}$} & \multirow{4}{*}{$\mathrm{m}$} & $1 \mathrm{H}_{6}$ & 1.83 & $1 \mathrm{H}$ & $\mathrm{m}$ & $1 \mathrm{H}_{6}$, & \multirow{2}{*}{1.92} & \multirow{2}{*}{$2 \mathrm{H}$} & \multirow{2}{*}{$\mathrm{m}$} \\
\hline & & & & $1 \mathrm{H}_{2}$ & 1.75 & $1 \mathrm{H}$ & $\mathrm{m}$ & $1 \mathrm{H}_{2}$ & & & \\
\hline & & & & $1 \mathrm{H}_{6}$ & 1.73 & $1 \mathrm{H}$ & $\mathrm{m}$ & \multirow{2}{*}{$\begin{array}{l}1 \mathrm{H}_{6}, \\
1 \mathrm{H}_{7}\end{array}$} & \multirow{2}{*}{1.77} & \multirow{2}{*}{$2 \mathrm{H}$} & \multirow{2}{*}{$\mathrm{m}$} \\
\hline & & & & $1 \mathrm{H}_{7}$ & 1.67 & $1 \mathrm{H}$ & $\mathrm{m}$ & & & & \\
\hline
\end{tabular}




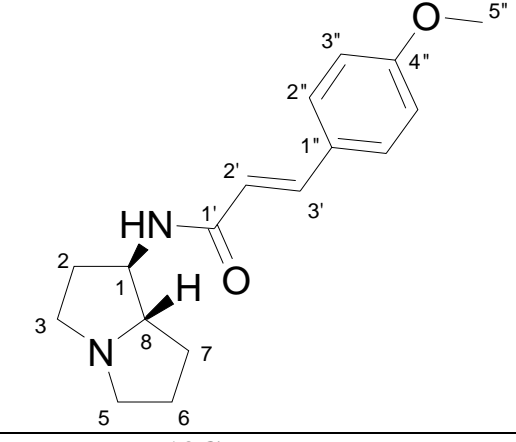

13C NMR data

\begin{tabular}{|c|c|c|c|c|c|}
\hline \multicolumn{6}{|c|}{ 13C NMR data } \\
\hline \multicolumn{2}{|c|}{ Our synthesis } & \multicolumn{2}{|c|}{$\begin{array}{c}\text { Ikhiri, Ahond and Poupat (50.3 } \\
\text { MHz) }\end{array}$} & \multicolumn{2}{|c|}{ Poupat (75 MHz) ${ }^{\text {s9 }}$} \\
\hline Attribution & Chem. Shift & Attribution & Chem. Shift & Attribution & Chem. Shift \\
\hline $\mathrm{C}_{1}$ & 166.2 & $\mathrm{C}_{1}$, & 166.3 & $\mathrm{C}_{1}$ & 166.0 \\
\hline $\mathrm{C}_{4}^{\prime \prime}$ & 160.9 & $\mathrm{C}_{4 \prime}$ & 161.0 & $\mathrm{C}_{4 \prime}$, & 160.5 \\
\hline $\mathrm{C}_{2}$ & 140.7 & $\mathrm{C}_{2}$ & 140.4 & $\mathrm{C}_{3}$ & 140.2 \\
\hline $\mathrm{C}_{2}$, & 129.4 & $\mathrm{C}_{2}$, & 129.3 & $\mathrm{C}_{2}$, & 129.0 \\
\hline $\mathrm{C}_{1}^{\prime \prime}$ & 127.6 & $\mathrm{C}_{1}$ & 127.9 & $\mathrm{C}_{1}$, & 127.3 \\
\hline $\mathrm{C}_{3}$, & 118.2 & $\mathrm{C}_{3}$, & 118.9 & $\mathrm{C}_{2}$ & 118.1 \\
\hline $\mathrm{C}_{3}$, & 114.2 & $\mathrm{C}_{3}$, & 114.4 & $\mathrm{C}_{3}$ & 113.8 \\
\hline $\mathrm{C}_{8}$ & 71.0 & $\mathrm{C}_{8}$ & 71.1 & $\mathrm{C}_{8}$ & 70.8 \\
\hline $\mathrm{C}_{5}$ & 55.43 & $\mathrm{C}_{5}$ & 55.7 & $\mathrm{C}_{5}$ & 55.0 \\
\hline $\mathrm{C}_{3}$ & 55.37 & \multirow{2}{*}{$\mathrm{C}_{1}$ and $\mathrm{C}_{3}$} & \multirow{2}{*}{55.3} & $\mathrm{C}_{3}$ & 54.9 \\
\hline $\mathrm{C}_{1}$ & 55.2 & & & $\mathrm{C}_{1}$ & 54.5 \\
\hline $\mathrm{C}_{5}$ & 53.4 & $\mathrm{C}_{5}$ & 53.4 & $\mathrm{C}_{5}$ & 53.0 \\
\hline $\mathrm{C}_{2}$ & 33.0 & $\mathrm{C}_{2}$ & 33.1 & $\mathrm{C}_{2}$ & 31.9 \\
\hline $\mathrm{C}_{7}$ & 30.7 & $\mathrm{C}_{7}$ & 30.7 & $\mathrm{C}_{7}$ & 30.1 \\
\hline $\mathrm{C}_{6}$ & 25.4 & $\mathrm{C}_{6}$ & 25.5 & $\mathrm{C}_{6}$ & 25.0 \\
\hline
\end{tabular}

${ }^{\mathrm{S} 8}$ Comparison data from isolation of absoulin, as disclosed by Ikhiri, Ahond and Poupat : Ikhiri, K.; Ahond, A.; Poupat, C.; Potier, P.; Pusset, J.; Sevene, T. J. Nat. Prod. 1987, 50, 626-630.

S9 Comparison data from synthesis of absoulin, as disclosed by Poupat: Christine, C.; Ikhiri, K.; Ahond, A.; Al Mourabit, A.; Poupat, C.; Potier, P. Tetrahedron 2000, 56, 18371850. 


\section{${ }^{1} \mathrm{H}$ NMR spectra of Absouline}
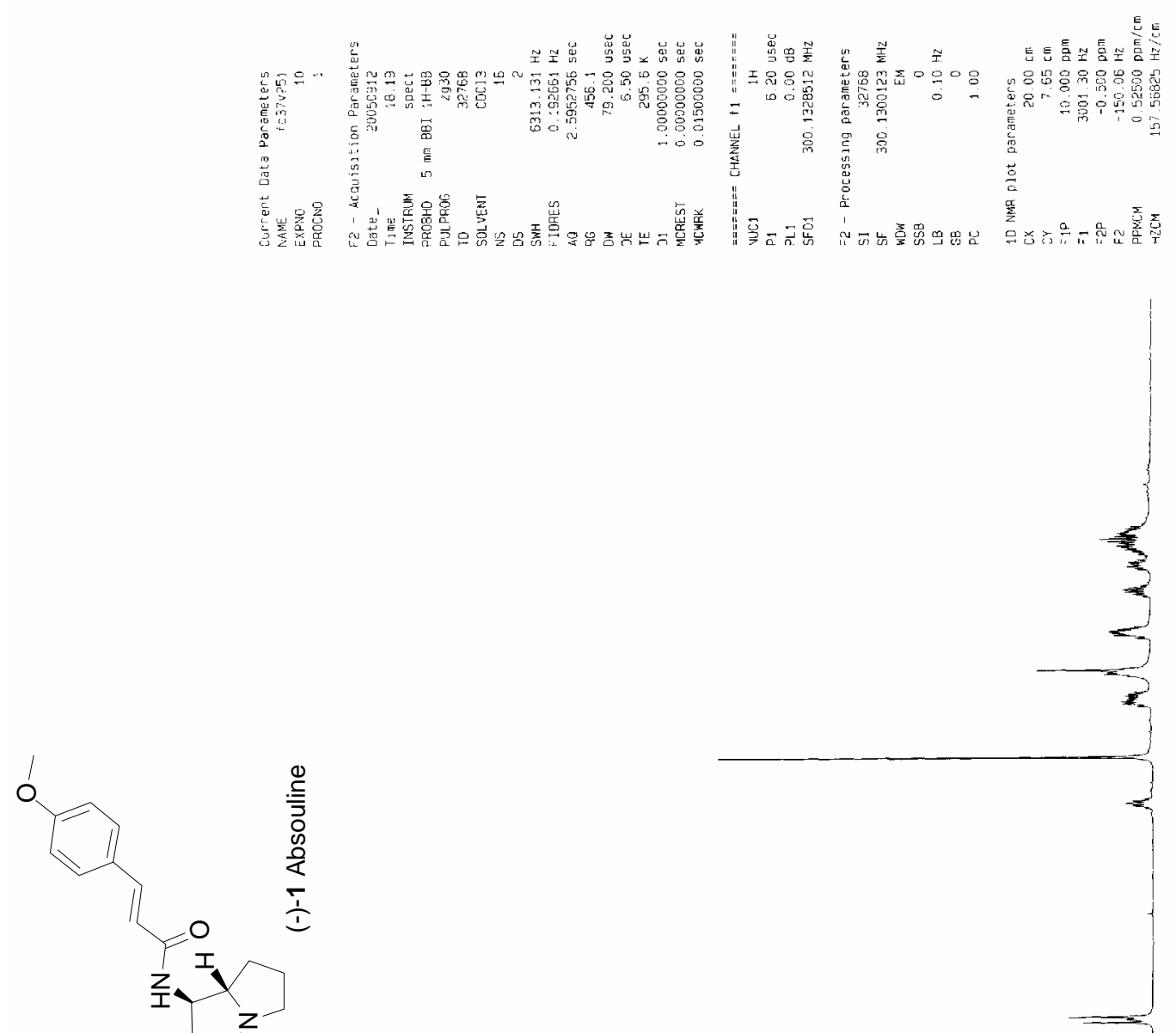


\section{${ }^{13} \mathrm{C}$ NMR spectra of Absouline}

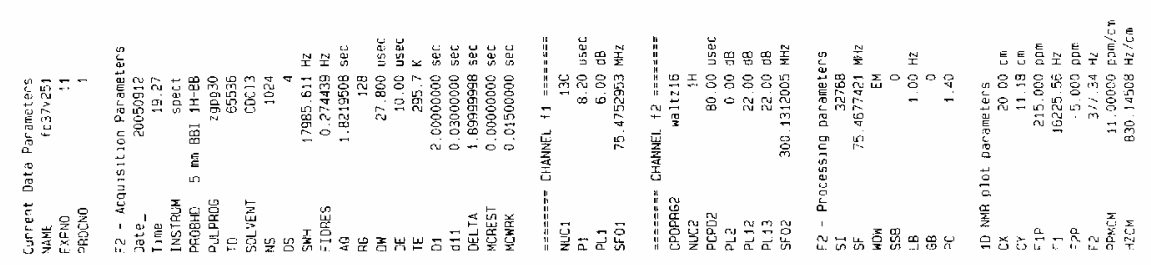

0

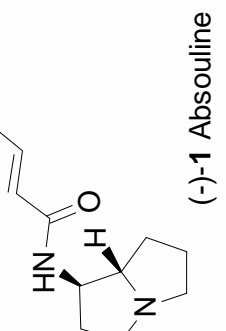

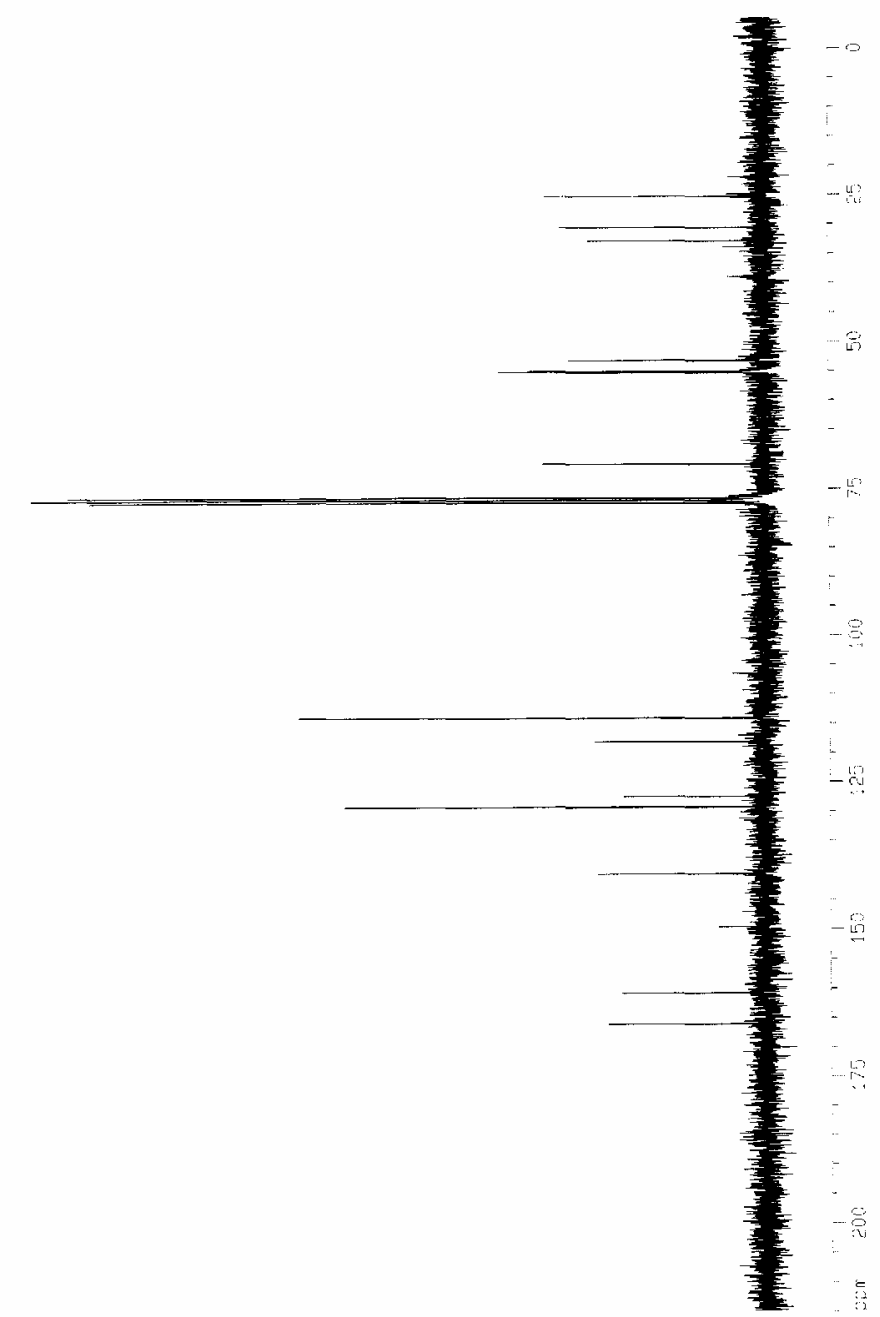




\section{Crystal data for $N$-Boc-5l}

Table 1. Crystal data and structure refinement for $\mathbf{N - B o c - 5 1 .}$

Identification code
Empirical formula
Formula weight
Temperature
Wavelength
Crystal system, space group
Unit cell dimensions

Volume

Z, Calculated density

Absorption coefficient

$F(\odot \odot \odot)$

crystal size

Theta range for data collection

Limiting indices

Reflections collected / unique

Completeness to theta $=25.00$

Absorption correction

Max. and min. transmission

Refinement method

Data / restraints / parameters

Goodness-of-fit on $F^{\wedge} 2$

Final $\mathrm{R}$ indices [I $>2 \mathrm{sigma}(\mathrm{I})]$

$\mathrm{R}$ indices (all data)

Absolute structure parameter

Largest diff. peak and hole
N-Boc- 51

C30 H36 N2 02

456.61

293(2) K

$0.71073 \mathrm{~A}$

Monoclinic, P2(1)

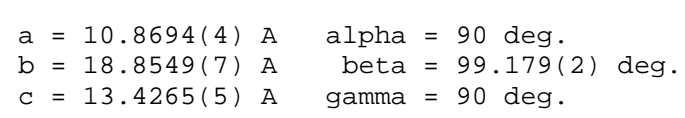

$2716.41(17) A^{\wedge} 3$

4, $1.116 \mathrm{Mg} / \mathrm{m}^{\wedge} 3$

๑. $069 \mathrm{~mm}^{\wedge}-1$

984

$0.50 \times 0.50 \times 0.35 \mathrm{~mm}$

1.54 to $25.00 \mathrm{deg}$.

$-12<=\mathrm{h}<=12, \quad-22<=\mathrm{k}<=22, \quad-15<=1<=15$

$196478 / 4942[\mathrm{R}($ int $)=0.0385]$

$100.0 \%$

Semi-empirical from equivalents

$\odot .9761$ and $\odot .9661$

Full-matrix least-squares on $F^{\wedge} 2$

$4942 / 1 / 625$

1.081

$R 1=0.0558, W R 2=0.1616$

$\mathrm{R} 1=0.0664, \quad W R 2=0.1792$

$\odot(10)$

0.303 and -0.324 e. $A^{\wedge}-3$ 
Table 2. Atomic coordinates $(x 10 \wedge 4)$ and equivalent isotropic displacement parameters $\left(A^{\wedge} 2 \times 10 \wedge 3\right)$ for $\mathbf{N - B o c - 5 1 .}$ $\mathrm{U}(\mathrm{eq})$ is defined as one third of the trace of the orthogonalized Uij tensor.

\begin{tabular}{|c|c|c|c|c|}
\hline & $x$ & $y$ & z & $\mathrm{U}(\mathrm{eq})$ \\
\hline$C(1 \mathrm{~A})$ & $-502(4)$ & $5456(3)$ & $-1322(3)$ & $71(1)$ \\
\hline$C(2 A)$ & $-849(5)$ & $6083(3)$ & $-1824(4)$ & $82(1)$ \\
\hline$C(3 A)$ & $-1869(5)$ & $6446(3)$ & $-1663(5)$ & $90(2)$ \\
\hline$C(4 A)$ & $-2528(7)$ & $6184(4)$ & $-981(8)$ & $132(3)$ \\
\hline$C(5 A)$ & $-2190(5)$ & $5556(3)$ & $-451(6)$ & $104(2)$ \\
\hline$C(6 A)$ & $-1174(3)$ & $5175(2)$ & $-637(3)$ & $52(1)$ \\
\hline$C(7 A)$ & $-854(3)$ & 4478(2) & $-103(2)$ & $48(1)$ \\
\hline$C(8 A)$ & $-1240(3)$ & $3810(2)$ & $-729(3)$ & $58(1)$ \\
\hline$N(9 A)$ & $-519(3)$ & $3260(2)$ & $-131(3)$ & $67(1)$ \\
\hline$C(10 A)$ & $713(3)$ & $3530(2)$ & $306(3)$ & 51(1) \\
\hline$C(11 A)$ & $1844(3)$ & $3306(2)$ & $-156(2)$ & $47(1)$ \\
\hline$C(12 A)$ & $1894(4)$ & $3379(2)$ & $-1184(3)$ & $63(1)$ \\
\hline$C(13 A)$ & $2956(4)$ & $3213(3)$ & $-1560(3)$ & $73(1)$ \\
\hline$C(14 A)$ & $4015(4)$ & $2977(3)$ & $-924(4)$ & $78(1)$ \\
\hline$C(15 A)$ & $3968(4)$ & 2899(3) & $83(4)$ & $76(1)$ \\
\hline$C(16 A)$ & $2894(4)$ & $3063(2)$ & $465(3)$ & $57(1)$ \\
\hline$C(17 A)$ & $543(3)$ & $4351(2)$ & $228(2)$ & $45(1)$ \\
\hline $\mathrm{N}(18 \mathrm{~A})$ & $1105(3)$ & $4723(2)$ & $1145(2)$ & $49(1)$ \\
\hline$C(19 A)$ & $553(4)$ & $4620(2)$ & $2057(3)$ & $54(1)$ \\
\hline$C(2 \odot A)$ & $-419(4)$ & $5159(2)$ & $2222(3)$ & $60(1)$ \\
\hline$C(21 A)$ & $-307(6)$ & $5869(3)$ & 1994(5) & $94(2)$ \\
\hline$C(22 A)$ & $-1209(7)$ & $6344(4)$ & $2170(6)$ & $123(2)$ \\
\hline$C(23 A)$ & $-2221(6)$ & $6128(4)$ & $2585(5)$ & $108(2)$ \\
\hline$C(24 A)$ & $-2330(5)$ & $5437(4)$ & $2803(5)$ & $97(2)$ \\
\hline$C(25 A)$ & $-1440(4)$ & $4955(3)$ & $2626(3)$ & $75(1)$ \\
\hline$C(26 A)$ & 2196(3) & $5081(2)$ & 1228(3) & 51(1) \\
\hline $0(27 A)$ & $2630(2)$ & $5102(2)$ & $342(2)$ & $62(1)$ \\
\hline$C(28 A)$ & $3783(4)$ & $5449(3)$ & $228(3)$ & $69(1)$ \\
\hline$C(29 A)$ & $-2633(4)$ & 3659(3) & $-864(4)$ & $82(1)$ \\
\hline$C(30 A)$ & $-582(5)$ & $2557(3)$ & $-554(6)$ & $97(2)$ \\
\hline $0(31 A)$ & $2706(3)$ & $5347(2)$ & $2005(2)$ & $72(1)$ \\
\hline$C(32 A)$ & $3628(13)$ & $6236(4)$ & $401(6)$ & $172(5)$ \\
\hline$C(33 A)$ & $4845(5)$ & $5107(8)$ & $939(6)$ & $177(6)$ \\
\hline$C(34 A)$ & $3901(5)$ & $5304(4)$ & $-860(4)$ & $90(2)$ \\
\hline$C(1 B)$ & $3084(4)$ & 7783(3) & $4640(4)$ & $83(1)$ \\
\hline$C(2 B)$ & $2727(6)$ & $7125(3)$ & $4232(6)$ & $104(2)$ \\
\hline$C(3 B)$ & $3259(6)$ & 6867(3) & 3441(5) & $95(2)$ \\
\hline$C(4 B)$ & $4155(6)$ & $7249(3)$ & $3078(4)$ & $92(2)$ \\
\hline$C(5 B)$ & $4493(5)$ & $7905(3)$ & $3484(3)$ & $76(1)$ \\
\hline$C(6 B)$ & $3952(3)$ & 8185(2) & $4251(3)$ & $54(1)$ \\
\hline$C(7 B)$ & $4320(3)$ & $8911(2)$ & $4689(3)$ & $52(1)$ \\
\hline$C(8 B)$ & $4093(3)$ & $9470(2)$ & $3911(2)$ & $51(1)$ \\
\hline$N(9 B)$ & $4776(4)$ & $10100(2)$ & $4278(3)$ & $83(1)$ \\
\hline$C(10 B)$ & $5942(4)$ & $9820(2)$ & $4989(3)$ & $52(1)$ \\
\hline$C(11 B)$ & $7165(3)$ & $10003(2)$ & $4664(3)$ & $52(1)$ \\
\hline$C(12 B)$ & $8138(4)$ & $10291(2)$ & $5330(3)$ & $68(1)$ \\
\hline$C(13 B)$ & $9273(5)$ & $10438(3)$ & $5037(5)$ & $92(2)$ \\
\hline$C(14 B)$ & $9443(5)$ & $10301(4)$ & $4067(6)$ & $102(2)$ \\
\hline$C(15 B)$ & $8484(6)$ & $10009(4)$ & $3388(4)$ & $94(2)$ \\
\hline$C(16 B)$ & $7359(4)$ & $9861(3)$ & $3687(3)$ & $74(1)$ \\
\hline$C(17 B)$ & $5727(3)$ & $9008(2)$ & $5025(2)$ & $45(1)$ \\
\hline$N(18 B)$ & $6233(3)$ & $8673(2)$ & $5991(2)$ & $47(1)$ \\
\hline$C(19 B)$ & $5650(4)$ & $8809(2)$ & $6881(3)$ & $53(1)$ \\
\hline$C(20 B)$ & 4746 (3) & $8247(2)$ & $7119(3)$ & $55(1)$ \\
\hline$C(21 B)$ & 4898(5) & $7539(3)$ & $6922(4)$ & $84(1)$ \\
\hline$C(22 B)$ & $4063(6)$ & $7041(3)$ & $7182(6)$ & $107(2)$ \\
\hline$C(23 B)$ & $3082(6)$ & $7238(4)$ & $7627(5)$ & $101(2)$ \\
\hline$C(24 B)$ & $2919(4)$ & $7929(4)$ & $7840(4)$ & $87(2)$ \\
\hline$C(25 B)$ & $3756(4)$ & $8435(3)$ & $7595(3)$ & $68(1)$ \\
\hline
\end{tabular}


3-Aminopyrrolidines via Ring Rearrangement of 2-Aminomethylazetidines. Synthesis of (-)-Absouline

$\begin{array}{lrrrr}C(26 B) & 7339(3) & 8317(2) & 6147(3) & 49(1) \\ \text { O(27B) } & 7827(2) & 8268(2) & 5291(2) & 62(1) \\ \text { C(28B) } & 8964(4) & 7867(3) & 5249(3) & 68(1) \\ \text { C(29B) } & 2774(5) & 9560(4) & 3483(5) & 102(2) \\ C(30 B) & 4060(6) & 10640(4) & 4813(7) & 121(3) \\ \text { O(31B) } & 7801(3) & 8078(2) & 6957(2) & 68(1) \\ C(32 B) & 8767(12) & 7104(4) & 5455(7) & 172(5) \\ C(33 B) & 10011(5) & 8201(8) & 5951(6) & 173(5) \\ C(34 B) & 9152(5) & 7969(4) & 4171(4) & 92(2)\end{array}$


Table 3. Bond lengths [A] and angles [deg] for $\mathbf{N - B o c - 5 1 .}$

\begin{tabular}{|c|c|}
\hline$C(1 A)-C(6 A)$ & $1.368(6)$ \\
\hline$C(1 A)-C(2 A)$ & $1.383(7)$ \\
\hline$C(1 A)-H(1 A)$ & 0.9300 \\
\hline$C(2 A)-C(3 A)$ & $1.350(8)$ \\
\hline$C(2 A)-H(2 A)$ & 0.9300 \\
\hline$C(3 A)-C(4 A)$ & $1.343(9)$ \\
\hline$C(3 A)-H(3 A)$ & 0.9300 \\
\hline$C(4 A)-C(5 A)$ & $1.400(9)$ \\
\hline$C(4 A)-H(4 A)$ & 0.9300 \\
\hline$C(5 A)-C(6 A)$ & $1.373(6)$ \\
\hline$C(5 A)-H(5 A)$ & 0.9300 \\
\hline$C(6 A)-C(7 A)$ & $1.510(5)$ \\
\hline$C(7 A)-C(17 A)$ & $1.532(4)$ \\
\hline$C(7 A)-C(8 A)$ & $1.534(5)$ \\
\hline$C(7 A)-H(7 A)$ & $\odot .9800$ \\
\hline$C(8 A)-N(9 A)$ & $1.461(5)$ \\
\hline$C(8 A)-C(29 A)$ & $1.524(5)$ \\
\hline$C(8 A)-H(8 A)$ & 0.9800 \\
\hline$N(9 A)-C(30 A)$ & $1.440(6)$ \\
\hline$N(9 A)-C(10 A)$ & $1.465(5)$ \\
\hline$C(10 A)-C(11 A)$ & $1.522(5)$ \\
\hline$C(10 A)-C(17 A)$ & $1.561(5)$ \\
\hline$C(10 A)-H(10 A)$ & $\odot .9800$ \\
\hline$C(11 A)-C(16 A)$ & $1.381(5)$ \\
\hline$C(11 \mathrm{~A})-C(12 \mathrm{~A})$ & $1.396(5)$ \\
\hline$C(12 A)-C(13 A)$ & $1.368(6)$ \\
\hline$C(12 A)-H(12 A)$ & 0.9300 \\
\hline$C(13 A)-C(14 A)$ & $1.393(7)$ \\
\hline$C(13 A)-H(13 A)$ & 0.9300 \\
\hline$C(14 A)-C(15 A)$ & $1.369(7)$ \\
\hline$C(14 A)-H(14 A)$ & $\odot .9300$ \\
\hline$C(15 A)-C(16 A)$ & $1.382(6)$ \\
\hline$C(15 A)-H(15 A)$ & 0.9300 \\
\hline$C(16 A)-H(16 A)$ & 0.9300 \\
\hline$C(17 A)-N(18 A)$ & $1.464(4)$ \\
\hline$C(17 A)-H(17 A)$ & 0.9800 \\
\hline$N(18 A)-C(26 A)$ & $1.355(5)$ \\
\hline$N(18 A)-C(19 A)$ & $1.460(4)$ \\
\hline$C(19 A)-C(20 A)$ & $1.508(6)$ \\
\hline$C(19 A)-H(19 A)$ & 0.9700 \\
\hline$C(19 A)-H(19 B)$ & $\odot .970 \odot$ \\
\hline$C(20 A)-C(25 A)$ & $1.366(6)$ \\
\hline$C(20 A)-C(21 A)$ & $1.383(7)$ \\
\hline$C(21 A)-C(22 A)$ & $1.376(8)$ \\
\hline$C(21 A)-H(21 A)$ & $\odot .9300$ \\
\hline$C(22 A)-C(23 A)$ & $1.371(10)$ \\
\hline$C(22 A)-H(22 A)$ & 0.9300 \\
\hline$C(23 A)-C(24 A)$ & $1.345(11)$ \\
\hline$C(23 A)-H(23 A)$ & $\odot .9300$ \\
\hline$C(24 A)-C(25 A)$ & $1.375(8)$ \\
\hline$C(24 A)-H(24 A)$ & 0.9300 \\
\hline$C(25 A)-H(25 A)$ & $\odot .9300$ \\
\hline$C(26 A)-0(31 A)$ & $1.209(4)$ \\
\hline$C(26 A)-0(27 A)$ & $1.348(4)$ \\
\hline $0(27 A)-C(28 A)$ & $1.443(4)$ \\
\hline$C(28 A)-C(34 A)$ & $1.511(7)$ \\
\hline$C(28 A)-C(32 A)$ & $1.515(10)$ \\
\hline$C(28 A)-C(33 A)$ & $1.520(8)$ \\
\hline$C(29 A)-H(29 A)$ & 0.9600 \\
\hline$C(29 A)-H(29 B)$ & $\odot .9600$ \\
\hline$C(29 A)-H(29 C)$ & 0.9600 \\
\hline$C(3 \odot A)-H(3 \odot A)$ & 0.9600 \\
\hline$C(30 A)-H(30 B)$ & 0.9600 \\
\hline$C(30 A)-H(30 C)$ & 0.9600 \\
\hline$C(32 A)-H(32 A)$ & 0.9600 \\
\hline$C(32 A)-H(32 B)$ & $\odot .960 \odot$ \\
\hline
\end{tabular}




\begin{tabular}{|c|c|}
\hline$C(32 A)-H(32 C)$ & 0.9600 \\
\hline$C(33 A)-H(33 A)$ & 0.9600 \\
\hline$C(33 A)-H(33 B)$ & 0.9600 \\
\hline$C(33 A)-H(33 C)$ & 0.9600 \\
\hline$C(34 A)-H(34 A)$ & 0.9600 \\
\hline$C(34 A)-H(34 B)$ & 0.9600 \\
\hline$C(34 A)-H(34 C)$ & 0.9600 \\
\hline$C(1 B)-C(6 B)$ & $1.377(6)$ \\
\hline$C(1 B)-C(2 B)$ & $1.386(8)$ \\
\hline$C(1 B)-H(1 B)$ & 0.9300 \\
\hline$C(2 B)-C(3 B)$ & $1.376(9)$ \\
\hline$C(2 B)-H(2 B)$ & 0.9300 \\
\hline$C(3 B)-C(4 B)$ & $1.363(9)$ \\
\hline$C(3 B)-H(3 B)$ & 0.9300 \\
\hline$C(4 B)-C(5 B)$ & $1.378(8)$ \\
\hline$C(4 B)-H(4 B)$ & 0.9300 \\
\hline$C(5 B)-C(6 B)$ & $1.370(6)$ \\
\hline$C(5 B)-H(5 B)$ & 0.9300 \\
\hline$C(6 B)-C(7 B)$ & $1.517(6)$ \\
\hline$C(7 B)-C(8 B)$ & $1.476(5)$ \\
\hline$C(7 B)-C(17 B)$ & $1.535(5)$ \\
\hline$C(7 B)-H(7 B)$ & $0.9800^{\circ}$ \\
\hline$C(8 B)-N(9 B)$ & $1.446(6)$ \\
\hline$C(8 B)-C(29 B)$ & $1.466(6)$ \\
\hline $\mathrm{C}(8 \mathrm{~B})-\mathrm{H}(8 \mathrm{~B})$ & 0.9800 \\
\hline$N(9 B)-C(30 B)$ & $1.529(7)$ \\
\hline$N(9 B)-C(10 B)$ & $1.553(5)$ \\
\hline$C(10 B)-C(11 B)$ & $1.503(5)$ \\
\hline$C(10 B)-C(17 B)$ & $1.551(5)$ \\
\hline$C(10 B)-H(10 B)$ & 0.9800 \\
\hline$C(11 B)-C(12 B)$ & $1.382(6)$ \\
\hline$C(11 B)-C(16 B)$ & $1.389(5)$ \\
\hline$C(12 B)-C(13 B)$ & $1.382(7)$ \\
\hline $\mathrm{C}(12 \mathrm{~B})-\mathrm{H}(12 \mathrm{~B})$ & 0.9300 \\
\hline$C(13 B)-C(14 B)$ & $1.369(9)$ \\
\hline$C(13 B)-H(13 B)$ & 0.9300 \\
\hline$C(14 B)-C(15 B)$ & $1.385(10)$ \\
\hline$C(14 B)-H(14 B)$ & 0.9300 \\
\hline$C(15 B)-C(16 B)$ & $1.374(7)$ \\
\hline$C(15 B)-H(15 B)$ & 0.9300 \\
\hline $\mathrm{C}(16 \mathrm{~B})-\mathrm{H}(16 \mathrm{~B})$ & 0.9300 \\
\hline $\mathrm{C}(17 \mathrm{~B})-\mathrm{N}(18 \mathrm{~B})$ & $1.469(4)$ \\
\hline$C(17 B)-H(17 B)$ & 0.9800 \\
\hline$N(18 B)-C(26 B)$ & $1.363(4)$ \\
\hline$N(18 B)-C(19 B)$ & $1.461(4)$ \\
\hline$C(19 B)-C(20 B)$ & $1.515(5)$ \\
\hline$C(19 B)-H(19 C)$ & $\odot .9700$ \\
\hline$C(19 B)-H(19 D)$ & 0.9700 \\
\hline$C(20 B)-C(21 B)$ & $1.376(7)$ \\
\hline$C(20 B)-C(25 B)$ & $1.382(5)$ \\
\hline$C(21 B)-C(22 B)$ & $1.388(7)$ \\
\hline$C(21 B)-H(21 B)$ & 0.9300 \\
\hline$C(22 B)-C(23 B)$ & $1.355(9)$ \\
\hline $\mathrm{C}(22 \mathrm{~B})-\mathrm{H}(22 \mathrm{~B})$ & 0.9300 \\
\hline$C(23 B)-C(24 B)$ & $1.351(10)$ \\
\hline$C(23 B)-H(23 B)$ & 0.9300 \\
\hline$C(24 B)-C(25 B)$ & $1.394(7)$ \\
\hline$C(24 B)-H(24 B)$ & 0.9300 \\
\hline$C(25 B)-H(25 B)$ & 0.9300 \\
\hline$C(26 B)-0(31 B)$ & $1.211(4)$ \\
\hline$C(26 B)-0(27 B)$ & $1.344(4)$ \\
\hline$O(27 \mathrm{~B})-\mathrm{C}(28 \mathrm{~B})$ & $1.457(4)$ \\
\hline$C(28 B)-C(32 B)$ & $1.487(10)$ \\
\hline$C(28 B)-C(33 B)$ & $1.496(9)$ \\
\hline$C(28 B)-C(34 B)$ & $1.506(7)$ \\
\hline$C(29 B)-H(29 D)$ & 0.9600 \\
\hline$C(29 B)-H(29 E)$ & 0.9600 \\
\hline$C(29 B)-H(29 F)$ & 0.9600 \\
\hline$C(30 B)-H(30 D)$ & 0.9600 \\
\hline$C(30 B)-H(30 E)$ & 0.9600 \\
\hline $\mathrm{C}(30 \mathrm{~B})-\mathrm{H}(30 \mathrm{~F})$ & 0.9600 \\
\hline
\end{tabular}




\begin{tabular}{|c|c|}
\hline$C(32 B)-H(32 D)$ & 0.9600 \\
\hline$C(32 B)-H(32 E)$ & 0.9600 \\
\hline$C(32 B)-H(32 F)$ & 0.9600 \\
\hline$C(33 B)-H(33 D)$ & 0.9600 \\
\hline$C(33 B)-H(33 E)$ & 0.9600 \\
\hline$C(33 B)-H(33 F)$ & 0.9600 \\
\hline$C(34 B)-H(34 D)$ & 0.9600 \\
\hline$C(34 B)-H(34 E)$ & 0.9600 \\
\hline$C(34 B)-H(34 F)$ & 0.9600 \\
\hline$C(6 A)-C(1 A)-C(2 A)$ & $121.7(4)$ \\
\hline$C(6 A)-C(1 A)-H(1 A)$ & 119.1 \\
\hline$C(2 A)-C(1 A)-H(1 A)$ & 119.1 \\
\hline$C(3 A)-C(2 A)-C(1 A)$ & $121.4(5)$ \\
\hline$C(3 A)-C(2 A)-H(2 A)$ & 119.3 \\
\hline$C(1 A)-C(2 A)-H(2 A)$ & 119.3 \\
\hline$C(4 A)-C(3 A)-C(2 A)$ & $117.7(5)$ \\
\hline$C(4 A)-C(3 A)-H(3 A)$ & 121.2 \\
\hline$C(2 A)-C(3 A)-H(3 A)$ & 121.2 \\
\hline$C(3 A)-C(4 A)-C(5 A)$ & $122.2(5)$ \\
\hline$C(3 A)-C(4 A)-H(4 A)$ & 118.9 \\
\hline$C(5 A)-C(4 A)-H(4 A)$ & 118.9 \\
\hline$C(6 A)-C(5 A)-C(4 A)$ & $120.2(5)$ \\
\hline$C(6 A)-C(5 A)-H(5 A)$ & 119.9 \\
\hline$C(4 A)-C(5 A)-H(5 A)$ & 119.9 \\
\hline$C(1 A)-C(6 A)-C(5 A)$ & $116.8(4)$ \\
\hline$C(1 A)-C(6 A)-C(7 A)$ & $123.3(3)$ \\
\hline$C(5 A)-C(6 A)-C(7 A)$ & $119.9(4)$ \\
\hline$C(6 A)-C(7 A)-C(17 A)$ & $114.7(3)$ \\
\hline$C(6 A)-C(7 A)-C(8 A)$ & $115.6(3)$ \\
\hline$C(17 A)-C(7 A)-C(8 A)$ & $101.6(3)$ \\
\hline$C(6 A)-C(7 A)-H(7 A)$ & 108.2 \\
\hline$C(17 A)-C(7 A)-H(7 A)$ & 108.2 \\
\hline$C(8 A)-C(7 A)-H(7 A)$ & 108.2 \\
\hline$N(9 A)-C(8 A)-C(29 A)$ & $111.6(4)$ \\
\hline$N(9 A)-C(8 A)-C(7 A)$ & $101.9(3)$ \\
\hline$C(29 A)-C(8 A)-C(7 A)$ & $113.5(4)$ \\
\hline$N(9 A)-C(8 A)-H(8 A)$ & 109.9 \\
\hline$C(29 A)-C(8 A)-H(8 A)$ & 109.9 \\
\hline$C(7 A)-C(8 A)-H(8 A)$ & 109.9 \\
\hline$C(30 A)-N(9 A)-C(8 A)$ & $116.8(4)$ \\
\hline$C(30 A)-N(9 A)-C(10 A)$ & $117.4(4)$ \\
\hline$C(8 A)-N(9 A)-C(10 A)$ & $110.5(3)$ \\
\hline$N(9 A)-C(10 A)-C(11 A)$ & $119.1(3)$ \\
\hline$N(9 A)-C(10 A)-C(17 A)$ & $103.1(3)$ \\
\hline$C(11 A)-C(10 A)-C(17 A)$ & $110.0(3)$ \\
\hline$N(9 A)-C(1 \odot A)-H(1 \odot A)$ & 108.1 \\
\hline$C(11 A)-C(10 A)-H(10 A)$ & 108.1 \\
\hline$C(17 A)-C(10 A)-H(10 A)$ & 108.1 \\
\hline$C(16 A)-C(11 A)-C(12 A)$ & $118.1(3)$ \\
\hline$C(16 A)-C(11 A)-C(10 A)$ & $119.3(3)$ \\
\hline$C(12 A)-C(11 A)-C(10 A)$ & $122.5(3)$ \\
\hline$C(13 A)-C(12 A)-C(11 A)$ & $120.7(4)$ \\
\hline$C(13 A)-C(12 A)-H(12 A)$ & 119.7 \\
\hline$C(11 A)-C(12 A)-H(12 A)$ & 119.7 \\
\hline$C(12 A)-C(13 A)-C(14 A)$ & $120.7(4)$ \\
\hline$C(12 A)-C(13 A)-H(13 A)$ & 119.6 \\
\hline$C(14 A)-C(13 A)-H(13 A)$ & 119.6 \\
\hline$C(15 A)-C(14 A)-C(13 A)$ & $118.8(4)$ \\
\hline$C(15 A)-C(14 A)-H(14 A)$ & 120.6 \\
\hline$C(13 A)-C(14 A)-H(14 A)$ & 120.6 \\
\hline$C\left(14 A^{\prime}\right)-C(15 A)-C(16 A)$ & $120.6(4)$ \\
\hline$C(14 A)-C(15 A)-H(15 A)$ & 119.7 \\
\hline$C\left(16 A^{\prime}\right)-C\left(15 A^{\prime}\right)-H\left(15 A^{\prime}\right)$ & 119.7 \\
\hline$C(11 A)-C(16 A)-C(15 A)$ & $121.1(4)$ \\
\hline$C(11 A)-C(16 A)-H(16 A)$ & 119.5 \\
\hline$C\left(15 A^{\prime}\right)-C\left(16 A^{\prime}\right)-H(16 A)$ & 119.5 \\
\hline$N(18 A)-C(17 A)-C(7 A)$ & $115.4(3)$ \\
\hline$N(18 A)-C(17 A)-C(10 A)$ & $112.9(3)$ \\
\hline$C(7 A)-C(17 A)-C(1 \odot A)$ & $106.1(3)$ \\
\hline $\mathrm{N}(18 \mathrm{~A})-\mathrm{C}(17 \mathrm{~A})-\mathrm{H}(17 \mathrm{~A})$ & 107.3 \\
\hline
\end{tabular}




\begin{tabular}{|c|c|}
\hline$C(7 A)-C(17 A)-H(17 A)$ & 107.3 \\
\hline$C(10 A)-C(17 A)-H(17 A)$ & 107.3 \\
\hline$C(26 A)-N(18 A)-C(19 A)$ & $118.0(3)$ \\
\hline$C(26 A)-N(18 A)-C(17 A)$ & $123.4(3)$ \\
\hline$C(19 A)-N(18 A)-C(17 A)$ & $118.1(3)$ \\
\hline$N(18 A)-C(19 A)-C(20 A)$ & $115.1(3)$ \\
\hline$N(18 A)-C(19 A)-H(19 A)$ & 108.5 \\
\hline$C(20 A)-C(19 A)-H(19 A)$ & 108.5 \\
\hline$N(18 A)-C(19 A)-H(19 B)$ & 108.5 \\
\hline$C(20 A)-C(19 A)-H(19 B)$ & 108.5 \\
\hline$H(19 A)-C(19 A)-H(19 B)$ & 107.5 \\
\hline$C(25 A)-C(20 A)-C(21 A)$ & $117.8(4)$ \\
\hline$C(25 A)-C(2 \odot A)-C(19 A)$ & $120.1(4)$ \\
\hline$C(21 A)-C(20 A)-C(19 A)$ & $122.1(4)$ \\
\hline$C(22 A)-C(21 A)-C(20 A)$ & $120.1(5)$ \\
\hline$C(22 A)-C(21 A)-H(21 A)$ & 120.0 \\
\hline$C(20 A)-C(21 A)-H(21 A)$ & 120.0 \\
\hline$C(23 A)-C(22 A)-C(21 A)$ & $121.1(7)$ \\
\hline$C(23 A)-C(22 A)-H(22 A)$ & 119.5 \\
\hline$C(21 A)-C(22 A)-H(22 A)$ & 119.5 \\
\hline$C(24 A)-C(23 A)-C(22 A)$ & $118.7(6)$ \\
\hline$C(24 A)-C(23 A)-H(23 A)$ & 120.7 \\
\hline$C(22 A)-C(23 A)-H(23 A)$ & 120.7 \\
\hline$C(23 A)-C(24 A)-C(25 A)$ & $120.9(5)$ \\
\hline$C(23 A)-C(24 A)-H(24 A)$ & 119.6 \\
\hline$C(25 A)-C(24 A)-H(24 A)$ & 119.5 \\
\hline$C(20 A)-C(25 A)-C(24 A)$ & $121.4(5)$ \\
\hline$C(20 A)-C(25 A)-H(25 A)$ & 119.3 \\
\hline$C(24 A)-C(25 A)-H(25 A)$ & 119.3 \\
\hline $0(31 A)-C(26 A)-0(27 A)$ & $124.9(3)$ \\
\hline $0(31 A)-C(26 A)-N(18 A)$ & $123.5(3)$ \\
\hline$O(27 A)-C(26 A)-N(18 A)$ & $111.5(3)$ \\
\hline$C(26 A)-0(27 A)-C(28 A)$ & $122.6(3)$ \\
\hline $0(27 A)-C(28 A)-C(34 A)$ & $103.3(3)$ \\
\hline$O(27 A)-C(28 A)-C(32 A)$ & $108.0(6)$ \\
\hline$C(34 A)-C(28 A)-C(32 A)$ & $110.8(5)$ \\
\hline $0(27 A)-C(28 A)-C(33 A)$ & $109.0(5)$ \\
\hline$C(34 A)-C(28 A)-C(33 A)$ & $110.9(6)$ \\
\hline$C(32 A)-C(28 A)-C(33 A)$ & $114.3(8)$ \\
\hline$C(8 A)-C(29 A)-H(29 A)$ & 109.5 \\
\hline$C(8 A)-C(29 A)-H(29 B)$ & 109.5 \\
\hline$H(29 A)-C(29 A)-H(29 B)$ & 109.5 \\
\hline$C(8 A)-C(29 A)-H(29 C)$ & 109.5 \\
\hline$H(29 A)-C(29 A)-H(29 C)$ & 109.5 \\
\hline$H(29 B)-C(29 A)-H(29 C)$ & 109.5 \\
\hline$N(9 A)-C(3 \odot A)-H(3 \odot A)$ & 109.5 \\
\hline$N(9 A)-C(30 A)-H(30 B)$ & 109.5 \\
\hline$H(30 A)-C(30 A)-H(30 B)$ & 109.5 \\
\hline$N(9 A)-C(30 A)-H(30 C)$ & 109.5 \\
\hline$H(30 A)-C(30 A)-H(30 C)$ & 109.5 \\
\hline$H(30 B)-C(30 A)-H(30 C)$ & 109.5 \\
\hline$C(28 A)-C(32 A)-H(32 A)$ & 109.5 \\
\hline$C(28 A)-C(32 A)-H(32 B)$ & 109.5 \\
\hline$H(32 A)-C(32 A)-H(32 B)$ & 109.5 \\
\hline$C(28 A)-C(32 A)-H(32 C)$ & 109.5 \\
\hline$H(32 A)-C(32 A)-H(32 C)$ & 109.5 \\
\hline$H(32 B)-C(32 A)-H(32 C)$ & 109.5 \\
\hline$C(28 A)-C(33 A)-H(33 A)$ & 109.5 \\
\hline$C(28 A)-C(33 A)-H(33 B)$ & 109.5 \\
\hline$H(33 A)-C(33 A)-H(33 B)$ & 109.5 \\
\hline$C(28 A)-C(33 A)-H(33 C)$ & 109.5 \\
\hline$H(33 A)-C(33 A)-H(33 C)$ & 109.5 \\
\hline$H(33 B)-C(33 A)-H(33 C)$ & 109.5 \\
\hline$C(28 A)-C(34 A)-H(34 A)$ & 109.5 \\
\hline$C(28 A)-C(34 A)-H(34 B)$ & 109.5 \\
\hline$H(34 A)-C(34 A)-H(34 B)$ & 109.5 \\
\hline$C(28 A)-C(34 A)-H(34 C)$ & 109.5 \\
\hline$H(34 A)-C(34 A)-H(34 C)$ & 109.5 \\
\hline$H(34 B)-C(34 A)-H(34 C)$ & 109.5 \\
\hline$C(6 B)-C(1 B)-C(2 B)$ & $120.6(5)$ \\
\hline$C(6 B)-C(1 B)-H(1 B)$ & 1107 \\
\hline
\end{tabular}




\begin{tabular}{|c|c|}
\hline$C(2 B)-C(1 B)-H(1 B)$ & 119.7 \\
\hline$C(3 B)-C(2 B)-C(1 B)$ & $119.7(5)$ \\
\hline$C(3 B)-C(2 B)-H(2 B)$ & 120.1 \\
\hline$C(1 B)-C(2 B)-H(2 B)$ & 120.1 \\
\hline$C(4 B)-C(3 B)-C(2 B)$ & $120.1(5)$ \\
\hline$C(4 B)-C(3 B)-H(3 B)$ & 120.0 \\
\hline$C(2 B)-C(3 B)-H(3 B)$ & 120.0 \\
\hline$C(3 B)-C(4 B)-C(5 B)$ & $119.5(5)$ \\
\hline$C(3 B)-C(4 B)-H(4 B)$ & 120.2 \\
\hline$C(5 B)-C(4 B)-H(4 B)$ & 120.2 \\
\hline$C(6 B)-C(5 B)-C(4 B)$ & $121.6(5)$ \\
\hline$C(6 B)-C(5 B)-H(5 B)$ & 119.2 \\
\hline$C(4 B)-C(5 B)-H(5 B)$ & 119.2 \\
\hline$C(5 B)-C(6 B)-C(1 B)$ & $118.4(4)$ \\
\hline$C(5 B)-C(6 B)-C(7 B)$ & $121.3(4)$ \\
\hline$C(1 B)-C(6 B)-C(7 B)$ & $120.3(4)$ \\
\hline$C(8 B)-C(7 B)-C(6 B)$ & $111.4(3)$ \\
\hline$C(8 B)-C(7 B)-C(17 B)$ & $99.8(3)$ \\
\hline$C(6 B)-C(7 B)-C(17 B)$ & $114.2(3)$ \\
\hline$C(8 B)-C(7 B)-H(7 B)$ & 110.3 \\
\hline$C(6 B)-C(7 B)-H(7 B)$ & 110.3 \\
\hline$C(17 B)-C(7 B)-H(7 B)$ & 110.3 \\
\hline$N(9 B)-C(8 B)-C(29 B)$ & $117.1(4)$ \\
\hline$N(9 B)-C(8 B)-C(7 B)$ & $109.1(3)$ \\
\hline$C(29 B)-C(8 B)-C(7 B)$ & $113.8(4)$ \\
\hline$N(9 B)-C(8 B)-H(8 B)$ & 105.3 \\
\hline$C(29 B)-C(8 B)-H(8 B)$ & 105.3 \\
\hline$C(7 B)-C(8 B)-H(8 B)$ & 105.3 \\
\hline$C(8 B)-N(9 B)-C(30 B)$ & $115.8(4)$ \\
\hline$C(8 B)-N(9 B)-C(10 B)$ & $104.8(3)$ \\
\hline$C(30 B)-N(9 B)-C(10 B)$ & $111.3(4)$ \\
\hline$C(11 B)-C(10 B)-C(17 B)$ & $112.4(3)$ \\
\hline$C(11 B)-C(10 B)-N(9 B)$ & $114.6(3)$ \\
\hline$C(17 B)-C(10 B)-N(9 B)$ & $104.0(3)$ \\
\hline$C(11 B)-C(10 B)-H(10 B)$ & 108.6 \\
\hline$C\left(17 B^{\prime}\right)-C(10 B)-H(10 B)$ & 108.6 \\
\hline$N(9 B)-C(10 B)-H(10 B)$ & 108.6 \\
\hline$C(12 B)-C(11 B)-C(16 B)$ & $118.1(4)$ \\
\hline$C(12 B)-C(11 B)-C(10 B)$ & $121.5(3)$ \\
\hline$C(16 B)-C(11 B)-C(10 B)$ & $120.3(3)$ \\
\hline$C(13 B)-C(12 B)-C(11 B)$ & $121.4(5)$ \\
\hline$C(13 B)-C(12 B)-H(12 B)$ & 119.3 \\
\hline$C(11 B)-C(12 B)-H(12 B)$ & 119.3 \\
\hline$C(14 B)-C(13 B)-C(12 B)$ & $119.7(5)$ \\
\hline$C(14 B)-C(13 B)-H(13 B)$ & 120.2 \\
\hline$C(12 B)-C(13 B)-H(13 B)$ & 120.2 \\
\hline$C(13 B)-C(14 B)-C(15 B)$ & $120.0(5)$ \\
\hline$C(13 B)-C(14 B)-H(14 B)$ & 120.0 \\
\hline$C(15 B)-C(14 B)-H(14 B)$ & 120.0 \\
\hline$C(16 B)-C(15 B)-C(14 B)$ & $120.0(5)$ \\
\hline$C(16 B)-C(15 B)-H(15 B)$ & 120.0 \\
\hline$C(14 B)-C(15 B)-H(15 B)$ & 120.0 \\
\hline$C(15 B)-C(16 B)-C(11 B)$ & $120.8(5)$ \\
\hline$C(15 B)-C(16 B)-H(16 B)$ & 119.6 \\
\hline$C(11 B)-C(16 B)-H(16 B)$ & 119.6 \\
\hline$N(18 B)-C(17 B)-C(7 B)$ & $115.0(3)$ \\
\hline$N(18 B)-C(17 B)-C(10 B)$ & $114.5(3)$ \\
\hline$C(7 B)-C(17 B)-C(10 B)$ & $104.9(3)$ \\
\hline $\mathrm{N}(18 \mathrm{~B})-\mathrm{C}(17 \mathrm{~B})-\mathrm{H}(17 \mathrm{~B})$ & 107.3 \\
\hline$C(7 B)-C(17 B)-H(17 B)$ & 107.3 \\
\hline$C(10 B)-C(17 B)-H(17 B)$ & 107.3 \\
\hline$C(26 B)-N(18 B)-C(19 B)$ & $116.6(3)$ \\
\hline$C(26 B)-N(18 B)-C(17 B)$ & $122.9(3)$ \\
\hline$C(19 B)-N(18 B)-C\left(17 B^{\prime}\right)$ & $119.8(3)$ \\
\hline$N(18 B)-C(19 B)-C(20 B)$ & $115.5(3)$ \\
\hline $\mathrm{N}(18 \mathrm{~B})-\mathrm{C}(19 \mathrm{~B})-\mathrm{H}(19 \mathrm{C})$ & 108.4 \\
\hline$C(20 B)-C(19 B)-H(19 C)$ & 108.4 \\
\hline$N(18 B)-C(19 B)-H(19 D)$ & 108.4 \\
\hline$C(20 B)-C(19 B)-H(19 D)$ & 108.4 \\
\hline$H(19 C)-C(19 B)-H(19 D)$ & 107.5 \\
\hline$C(21 B)-C(20 B)-C(25 B)$ & $117.7(4)$ \\
\hline
\end{tabular}




\begin{tabular}{|c|c|}
\hline$C(21 B)-C(20 B)-C(19 B)$ & $122.4(4)$ \\
\hline$C(25 B)-C(20 B)-C(19 B)$ & $119.8(4)$ \\
\hline$C(20 B)-C(21 B)-C(22 B)$ & $120.3(5)$ \\
\hline$C(20 B)-C(21 B)-H(21 B)$ & 119.9 \\
\hline$C(22 B)-C(21 B)-H(21 B)$ & 119.9 \\
\hline$C(23 B)-C(22 B)-C(21 B)$ & $121.2(6)$ \\
\hline$C(23 B)-C(22 B)-H(22 B)$ & 119.4 \\
\hline$C(21 B)-C(22 B)-H(22 B)$ & 119.4 \\
\hline$C(24 B)-C(23 B)-C(22 B)$ & $119.8(5$ \\
\hline$C(24 B)-C(23 B)-H(23 B)$ & 120.1 \\
\hline $\mathrm{C}(22 \mathrm{~B})-\mathrm{C}(23 \mathrm{~B})-\mathrm{H}(23 \mathrm{~B})$ & 120.1 \\
\hline$C(23 B)-C(24 B)-C(25 B)$ & $119.8(5)$ \\
\hline$C(23 B)-C(24 B)-H(24 B)$ & 120.1 \\
\hline$C(25 B)-C(24 B)-H(24 B)$ & 120.1 \\
\hline$C(20 B)-C(25 B)-C(24 B)$ & $121.3(5)$ \\
\hline$C(20 B)-C(25 B)-H(25 B)$ & 119.4 \\
\hline$C(24 B)-C(25 B)-H(25 B)$ & 119.4 \\
\hline $0(31 B)-C(26 B)-0(27 B)$ & $125.3(3)$ \\
\hline$O(31 B)-C(26 B)-N(18 B)$ & $123.4(3)$ \\
\hline$O(27 B)-C(26 B)-N(18 B)$ & $111.2(3)$ \\
\hline$C(26 B)-0(27 B)-C(28 B)$ & $121.5(3)$ \\
\hline$O(27 B)-C(28 B)-C(32 B)$ & $110.3(6)$ \\
\hline$O(27 B)-C(28 B)-C(33 B)$ & $108.8(5)$ \\
\hline$C(32 B)-C(28 B)-C(33 B)$ & $114.1(8)$ \\
\hline$O(27 B)-C(28 B)-C(34 B)$ & $102.5(3)$ \\
\hline$C(32 B)-C(28 B)-C(34 B)$ & $110.4(5)$ \\
\hline$C(33 B)-C(28 B)-C(34 B)$ & $110.2(6)$ \\
\hline$C(8 B)-C(29 B)-H(29 D)$ & 109.5 \\
\hline$C(8 B)-C(29 B)-H(29 E)$ & 109.5 \\
\hline$H(29 D)-C(29 B)-H(29 E)$ & 109.5 \\
\hline$C(8 B)-C(29 B)-H(29 F)$ & 109.5 \\
\hline$H(29 D)-C(29 B)-H(29 F)$ & 109.5 \\
\hline$H(29 E)-C(29 B)-H(29 F)$ & 109.5 \\
\hline$N(9 B)-C(30 B)-H(30 D)$ & 109.5 \\
\hline$N(9 B)-C(30 B)-H(30 E)$ & 109.5 \\
\hline$H(3 \odot D)-C(3 \odot B)-H(3 \odot E)$ & 109.5 \\
\hline$N(9 B)-C(30 B)-H(30 F)$ & 109.5 \\
\hline$H(30 D)-C(30 B)-H(30 F)$ & 109.5 \\
\hline$H(30 E)-C(30 B)-H(30 F)$ & 109.5 \\
\hline$C(28 B)-C(32 B)-H(32 D)$ & 109.5 \\
\hline$C(28 B)-C(32 B)-H(32 E)$ & 109.5 \\
\hline$H(32 D)-C(32 B)-H(32 E)$ & 109.5 \\
\hline$C(28 B)-C(32 B)-H(32 F)$ & 109.5 \\
\hline$H(32 D)-C(32 B)-H(32 F)$ & 109.5 \\
\hline $\mathrm{H}(32 \mathrm{E})-\mathrm{C}(32 \mathrm{~B})-\mathrm{H}(32 \mathrm{~F})$ & 109.5 \\
\hline$C(28 B)-C(33 B)-H(33 D)$ & 109.5 \\
\hline$C(28 B)-C(33 B)-H(33 E)$ & 109.5 \\
\hline$H(33 D)-C(33 B)-H(33 E)$ & 109.5 \\
\hline$C(28 B)-C(33 B)-H(33 F)$ & 109.5 \\
\hline$H(33 D)-C(33 B)-H(33 F)$ & 109.5 \\
\hline$H(33 E)-C(33 B)-H(33 F)$ & 109.5 \\
\hline$C(28 B)-C(34 B)-H(34 D)$ & 109.5 \\
\hline$C(28 B)-C(34 B)-H(34 E)$ & 109.5 \\
\hline$H(34 D)-C(34 B)-H(34 E)$ & 109.5 \\
\hline$C(28 B)-C(34 B)-H(34 F)$ & 109.5 \\
\hline$H(34 D)-C(34 B)-H(34 F)$ & 109.5 \\
\hline $\mathrm{H}(34 \mathrm{E})-\mathrm{C}(34 \mathrm{~B})-\mathrm{H}(34 \mathrm{~F})$ & 109.5 \\
\hline
\end{tabular}


Table 4. Anisotropic displacement parameters $\left(A^{\wedge} 2 \times 10 \wedge 3\right)$ for $\mathbf{N - B o c - 5 1 .}$ The anisotropic displacement factor exponent takes the form: $-2 \mathrm{pi} \wedge 2\left[\mathrm{~h}^{\wedge} 2 \mathrm{a}^{\star \wedge} 2 \mathrm{U} 11+\ldots+2 \mathrm{hk} \mathrm{a}^{\star} \mathrm{b}^{\star} \mathrm{U} 12\right]$

\begin{tabular}{|c|c|c|c|c|c|c|}
\hline & U11 & U22 & U33 & U23 & U13 & U12 \\
\hline$C(1 A)$ & $82(3)$ & $76(3)$ & $62(2)$ & $20(2)$ & $29(2)$ & $23(2)$ \\
\hline$C(2 A)$ & $105(4)$ & $80(3)$ & $65(2)$ & $17(2)$ & $23(2)$ & $13(3)$ \\
\hline$C(3 A)$ & $98(4)$ & $64(3)$ & $106(4)$ & $16(3)$ & $11(3)$ & $21(3)$ \\
\hline$C(4 A)$ & $101(4)$ & $92(4)$ & $220(8)$ & $51(5)$ & $77(5)$ & $48(4)$ \\
\hline$C(5 A)$ & $82(3)$ & $82(3)$ & $162(6)$ & $37(4)$ & $65(4)$ & $27(3)$ \\
\hline$C(6 A)$ & $44(2)$ & $56(2)$ & $55(2)$ & $-2(2)$ & $5(1)$ & $0(2)$ \\
\hline$C(7 A)$ & $42(2)$ & $55(2)$ & $47(2)$ & $-1(2)$ & $9(1)$ & $-5(1)$ \\
\hline$C(8 A)$ & $49(2)$ & $57(2)$ & $66(2)$ & $-3(2)$ & $7(2)$ & $-8(2)$ \\
\hline$N(9 A)$ & $58(2)$ & $50(2)$ & $88(2)$ & $1(2)$ & $2(2)$ & $-11(1)$ \\
\hline$C(10 A)$ & $51(2)$ & $47(2)$ & $54(2)$ & $1(1)$ & $8(1)$ & $-4(1)$ \\
\hline$C(11 A)$ & $50(2)$ & $43(2)$ & $47(2)$ & $-9(1)$ & 4(1) & $-1(1)$ \\
\hline$C(12 A)$ & $65(2)$ & $74(3)$ & $48(2)$ & $-3(2)$ & $2(2)$ & $\Theta(2)$ \\
\hline$C(13 A)$ & $79(3)$ & $89(3)$ & $56(2)$ & $-17(2)$ & $20(2)$ & $-10(2)$ \\
\hline$C(14 A)$ & $63(2)$ & $91(3)$ & $84(3)$ & $-25(3)$ & $23(2)$ & $-1(2)$ \\
\hline$C(15 A)$ & $56(2)$ & $84(3)$ & $82(3)$ & $-16(2)$ & $-2(2)$ & $12(2)$ \\
\hline$C(16 A)$ & $60(2)$ & $58(2)$ & $50(2)$ & $-7(2)$ & $-2(2)$ & $3(2)$ \\
\hline$C(17 A)$ & $44(2)$ & $46(2)$ & $43(2)$ & $-1(1)$ & $6(1)$ & $-4(1)$ \\
\hline$N(18 A)$ & $51(2)$ & $51(2)$ & $45(1)$ & $-4(1)$ & $9(1)$ & $-6(1)$ \\
\hline$C(19 A)$ & $65(2)$ & $53(2)$ & $45(2)$ & $\odot(2)$ & $13(2)$ & $-4(2)$ \\
\hline$C(20 A)$ & $68(2)$ & $63(2)$ & $51(2)$ & $-12(2)$ & $16(2)$ & $-8(2)$ \\
\hline$C(21 A)$ & $114(4)$ & $61(3)$ & $121(4)$ & $-5(3)$ & $65(3)$ & $4(3)$ \\
\hline$c(22 A)$ & $139(6)$ & $74(4)$ & $170(7)$ & $-14(4)$ & $69(5)$ & $13(4)$ \\
\hline$C(23 A)$ & $92(4)$ & $110(5)$ & $126(5)$ & $-42(4)$ & $29(3)$ & $19(4)$ \\
\hline$C(24 A)$ & $67(3)$ & $129(6)$ & $99(4)$ & $-26(4)$ & $29(3)$ & $-6(3)$ \\
\hline$C(25 A)$ & $66(2)$ & $88(3)$ & $74(3)$ & $-5(2)$ & $20(2)$ & $-11(2)$ \\
\hline$C(26 A)$ & $52(2)$ & $48(2)$ & $5 \odot(2)$ & $-4(2)$ & $4(2)$ & $-3(2)$ \\
\hline $0(27 A)$ & $54(1)$ & $74(2)$ & $6 \odot(1)$ & $-11(1)$ & $14(1)$ & $-22(1)$ \\
\hline$C(28 A)$ & $58(2)$ & $77(3)$ & $70(2)$ & $8(2)$ & $8(2)$ & $-28(2)$ \\
\hline$C(29 A)$ & $52(2)$ & $88(3)$ & $102(3)$ & $-4(3)$ & $-4(2)$ & $-20(2)$ \\
\hline$C(3 \odot A)$ & $84(3)$ & $57(3)$ & $143(5)$ & $-17(3)$ & $-4(3)$ & $-16(2)$ \\
\hline $0(31 A)$ & $74(2)$ & $78(2)$ & $61(2)$ & $-15(2)$ & $3(1)$ & $-22(2)$ \\
\hline$C(32 A)$ & $331(15)$ & $81(5)$ & $123(5)$ & $-20(4)$ & $93(7)$ & $-88(7)$ \\
\hline$C(33 A)$ & $50(3)$ & $353(16)$ & $126(5)$ & $104(8)$ & $12(3)$ & $7(5)$ \\
\hline$C(34 A)$ & $84(3)$ & $107(4)$ & $85(3)$ & $3(3)$ & $32(2)$ & $-28(3)$ \\
\hline$C(1 B)$ & $66(2)$ & $85(3)$ & $103(3)$ & $-19(3)$ & $27(2)$ & $-11(2)$ \\
\hline$C(2 B)$ & $94(4)$ & $83(4)$ & $138(5)$ & $-17(4)$ & $31(4)$ & $-33(3)$ \\
\hline$C(3 B)$ & $114(4)$ & $63(3)$ & $102(4)$ & $-19(3)$ & $4(3)$ & $-23(3)$ \\
\hline$C(4 B)$ & $136(5)$ & $71(3)$ & $70(3)$ & $-19(2)$ & $24(3)$ & $-17(3)$ \\
\hline$C(5 B)$ & $103(3)$ & $74(3)$ & $56(2)$ & $-10(2)$ & $22(2)$ & $-18(3)$ \\
\hline$C(6 B)$ & $50(2)$ & $59(2)$ & $52(2)$ & $1(2)$ & $\odot(1)$ & $\odot(2)$ \\
\hline$C(7 B)$ & $50(2)$ & $53(2)$ & $54(2)$ & $-1(2)$ & $11(1)$ & $9(2)$ \\
\hline$C(8 B)$ & $51(2)$ & $55(2)$ & $46(2)$ & $6(2)$ & $-1(1)$ & $16(2)$ \\
\hline$N(9 B)$ & $88(3)$ & $72(2)$ & $90(3)$ & $15(2)$ & $13(2)$ & $23(2)$ \\
\hline$C(10 B)$ & $68(2)$ & $46(2)$ & $45(2)$ & $2(1)$ & $14(2)$ & $7(2)$ \\
\hline$C(11 B)$ & $62(2)$ & $44(2)$ & $48(2)$ & $9(1)$ & $7(2)$ & $2(2)$ \\
\hline$C(12 B)$ & $78(3)$ & $56(2)$ & $65(2)$ & $14(2)$ & $-2(2)$ & $-5(2)$ \\
\hline$C(13 B)$ & $65(3)$ & $87(4)$ & $117(4)$ & $37(3)$ & $-7(3)$ & $-17(2)$ \\
\hline$C(14 B)$ & $67(3)$ & $104(4)$ & $141(5)$ & $52(4)$ & $38(3)$ & $8(3)$ \\
\hline$C(15 B)$ & $100(4)$ & $102(4)$ & $91(3)$ & 18(3) & $50(3)$ & $-3(3)$ \\
\hline$C(16 B)$ & $82(3)$ & $82(3)$ & $61(2)$ & $3(2)$ & $22(2)$ & $-12(2)$ \\
\hline$C(17 B)$ & $48(2)$ & $49(2)$ & $39(1)$ & $2(1)$ & $7(1)$ & $6(1)$ \\
\hline$N(18 B)$ & $51(1)$ & $47(2)$ & $42(1)$ & $2(1)$ & $6(1)$ & $5(1)$ \\
\hline$C(19 B)$ & $64(2)$ & $51(2)$ & $46(2)$ & $-1(2)$ & $13(2)$ & $4(2)$ \\
\hline$C(20 B)$ & $60(2)$ & $59(2)$ & $46(2)$ & $8(2)$ & $8(1)$ & $6(2)$ \\
\hline$C(21 B)$ & $95(3)$ & $54(2)$ & $109(4)$ & $9(2)$ & $40(3)$ & $-3(2)$ \\
\hline$C(22 B)$ & $114(4)$ & $64(3)$ & $151(6)$ & $19(4)$ & $46(4)$ & $-8(3)$ \\
\hline$C(23 B)$ & $87(4)$ & $101(5)$ & $115(4)$ & $41(4)$ & $20(3)$ & $-20(3)$ \\
\hline$C(24 B)$ & $63(2)$ & $120(5)$ & $79(3)$ & $14(3)$ & $14(2)$ & $-3(3)$ \\
\hline$C(25 B)$ & $56(2)$ & $86(3)$ & $63(2)$ & $2(2)$ & $12(2)$ & $4(2)$ \\
\hline$C(26 B)$ & $52(2)$ & $45(2)$ & $49(2)$ & 1(2) & 4(1) & 1(2) \\
\hline $0(27 B)$ & $56(1)$ & $72(2)$ & $59(1)$ & $11(1)$ & $14(1)$ & $22(1)$ \\
\hline
\end{tabular}




\begin{tabular}{rccrrrr}
$\mathrm{C}(28 \mathrm{~B})$ & $68(2)$ & $73(3)$ & $62(2)$ & $-6(2)$ & $5(2)$ & $32(2)$ \\
$\mathrm{C}(29 \mathrm{~B})$ & $81(3)$ & $97(4)$ & $113(4)$ & $5(3)$ & $-29(3)$ & $26(3)$ \\
$\mathrm{C}(30 \mathrm{~B})$ & $99(4)$ & $81(4)$ & $177(7)$ & $-32(4)$ & $4(4)$ & $38(3)$ \\
$\mathrm{O}(31 \mathrm{~B})$ & $67(2)$ & $79(2)$ & $52(1)$ & $11(1)$ & $-3(1)$ & $16(1)$ \\
$\mathrm{C}(32 \mathrm{~B})$ & $316(14)$ & $75(4)$ & $150(7)$ & $23(4)$ & $111(8)$ & $78(7)$ \\
$\mathrm{C}(33 \mathrm{~B})$ & $57(3)$ & $339(16)$ & $121(5)$ & $-96(8)$ & $6(3)$ & $24(5)$ \\
$\mathrm{C}(34 \mathrm{~B})$ & $86(3)$ & $119(5)$ & $75(3)$ & $-3(3)$ & $25(2)$ & $34(3)$ \\
\hline
\end{tabular}

Table 5. Hydrogen coordinates $(x 10 \wedge 4)$ and isotropic displacement parameters $\left(A^{\wedge} 2 \times 10 \wedge 3\right)$ for $\boldsymbol{N}$-Boc-5l.

\begin{tabular}{|c|c|c|c|c|}
\hline & $x$ & $y$ & z & $\mathrm{U}(\mathrm{eq})$ \\
\hline$H(1 A)$ & 206 & 5220 & -1452 & 86 \\
\hline $\mathrm{H}(2 \mathrm{~A})$ & -368 & 6259 & -2283 & 99 \\
\hline$H(3 A)$ & -2108 & 6863 & -2011 & 108 \\
\hline$H(4 A)$ & -3233 & 6427 & -858 & 159 \\
\hline$H\left(5 A^{\prime}\right)$ & -2654 & 5396 & 29 & 124 \\
\hline $\mathrm{H}(7 \mathrm{~A})$ & -1259 & 4466 & 499 & 58 \\
\hline$H(8 A)$ & -972 & 3849 & -1390 & 69 \\
\hline $\mathrm{H}(10 \mathrm{~A})$ & 867 & 3402 & 1023 & 61 \\
\hline $\mathrm{H}(12 \mathrm{~A})$ & 1198 & 3542 & -1617 & 76 \\
\hline$H(13 A)$ & 2971 & 3259 & -2247 & 88 \\
\hline$H(14 A)$ & 4742 & 2875 & -1178 & 94 \\
\hline$H(15 A)$ & 4664 & 2734 & 514 & 91 \\
\hline$H(16 A)$ & 2879 & 3008 & 1151 & 68 \\
\hline $\mathrm{H}(17 \mathrm{~A})$ & 969 & 4514 & -321 & 53 \\
\hline$H(19 A)$ & 1214 & 4630 & 2635 & 65 \\
\hline$H(19 B)$ & 177 & 4153 & 2031 & 65 \\
\hline $\mathrm{H}(21 \mathrm{~A})$ & 379 & 6026 & 1721 & 112 \\
\hline$H(22 A)$ & -1132 & 6819 & 2006 & 147 \\
\hline$H(23 A)$ & -2821 & 6453 & 2712 & 130 \\
\hline$H(24 A)$ & -3017 & 5282 & 3077 & 116 \\
\hline$H(25 A)$ & -1534 & 4481 & 2785 & 90 \\
\hline $\mathrm{H}(29 \mathrm{~A})$ & -2811 & 3228 & -1240 & 124 \\
\hline$H(29 B)$ & -3081 & $4 \odot 45$ & -1222 & 124 \\
\hline $\mathrm{H}(29 \mathrm{C})$ & -2886 & 3610 & -214 & 124 \\
\hline$H(3 \odot A)$ & -1394 & 2360 & -538 & 145 \\
\hline $\mathrm{H}(30 \mathrm{~B})$ & 38 & 2262 & -165 & 145 \\
\hline $\mathrm{H}(30 \mathrm{C})$ & -433 & 2578 & -1239 & 145 \\
\hline $\mathrm{H}(32 \mathrm{~A})$ & 2974 & 6419 & -97 & 258 \\
\hline$H(32 B)$ & 4394 & 6477 & 349 & 258 \\
\hline $\mathrm{H}(32 \mathrm{C})$ & 3420 & 6310 & 1062 & 258 \\
\hline$H(33 A)$ & 4681 & 5139 & 1619 & 265 \\
\hline$H(33 B)$ & 5610 & 5348 & 887 & 265 \\
\hline $\mathrm{H}(33 \mathrm{C})$ & 4915 & 4617 & 760 & 265 \\
\hline$H(34 A)$ & 3928 & 4801 & -967 & 135 \\
\hline$H(34 B)$ & 4654 & 5516 & -1010 & 135 \\
\hline$H(34 C)$ & 3198 & 5502 & -1294 & 135 \\
\hline $\mathrm{H}(1 \mathrm{~B})$ & 2734 & 7954 & 5182 & 100 \\
\hline $\mathrm{H}(2 \mathrm{~B})$ & 2131 & 6859 & 4492 & 125 \\
\hline $\mathrm{H}(3 \mathrm{~B})$ & 3007 & 6431 & 3155 & 113 \\
\hline $\mathrm{H}(4 \mathrm{~B})$ & 4536 & 7068 & 2559 & 110 \\
\hline$H(5 B)$ & 5103 & 8164 & 3232 & 92 \\
\hline$H(7 B)$ & 3869 & 9017 & 5247 & 62 \\
\hline $\mathrm{H}(8 \mathrm{~B})$ & 4493 & 9299 & 3352 & 62 \\
\hline
\end{tabular}


3-Aminopyrrolidines via Ring Rearrangement of 2-Aminomethylazetidines. Synthesis of (-)-Absouline

\begin{tabular}{lrrrr}
$H(10 B)$ & 5938 & 10015 & 5666 & 63 \\
$H(12 B)$ & 8026 & 10388 & 5989 & 81 \\
$H(13 B)$ & 9919 & 10630 & 5497 & 110 \\
$H(14 B)$ & 10202 & 10404 & 3864 & 122 \\
$H(15 B)$ & 8601 & 9913 & 2730 & 113 \\
$H(16 B)$ & 6720 & 9662 & 3228 & 89 \\
$H(17 B)$ & 6148 & 8792 & 4507 & 54 \\
$H(19 C)$ & 5211 & 9258 & 6787 & 64 \\
$H(19 D)$ & 6303 & 8860 & 7460 & 64 \\
$H(21 B)$ & 5563 & 7393 & 6613 & 100 \\
$H(22 B)$ & 4180 & 6564 & 7049 & 128 \\
$H(23 B)$ & 2523 & 6899 & 7786 & 121 \\
$H(24 B)$ & 2250 & 8066 & 8150 & 104 \\
$H(25 B)$ & 3646 & 8908 & 7754 & 82 \\
$H(29 D)$ & 2315 & 9692 & 4008 & 153 \\
$H(29 E)$ & 2452 & 9122 & 3184 & 153 \\
$H(29 F)$ & 2694 & 9924 & 2978 & 153 \\
$H(30 D)$ & 3427 & 10859 & 4330 & 181 \\
$H(30 E)$ & 4625 & 10996 & 5125 & 181 \\
$H(30 F)$ & 3679 & 10404 & 5319 & 181 \\
$H(32 D)$ & 8617 & 7049 & 6136 & 259 \\
$H(32 E)$ & 9495 & 6839 & 5364 & 259 \\
$H(32 F)$ & 8061 & 6931 & 4997 & 259 \\
$H(33 D)$ & 10061 & 8695 & 5791 & 260 \\
$H(33 E)$ & 10780 & 7971 & 5878 & 260 \\
$H(33 F)$ & 9863 & 8150 & 6633 & 260 \\
$H(34 D)$ & 8437 & 7794 & 3726 & 138 \\
$H(34 E)$ & 9880 & 7713 & 4055 & 138 \\
$H(34 F)$ & 9260 & 8464 & 4045 & 138 \\
\hline
\end{tabular}

